\title{
Large Deviations for Gibbs Measures with Singular Hamiltonians and Emergence of Kähler-Einstein Metrics
}

\author{
Robert J. Berman \\ Chalmers tekniska hogskola, Göteborg, Sweden. E-mail: robertb@chalmers.se
}

Received: 4 October 2016 / Accepted: 5 May 2017

Published online: 17 June 2017 - (C) The Author(s) 2017. This article is an open access publication

\begin{abstract}
In the present paper and the companion paper (Berman, Kähler-Einstein metrics, canonical random point processes and birational geometry. arXiv:1307.3634, 2015) a probabilistic (statistical-mechanical) approach to the construction of canonical metrics on complex algebraic varieties $X$ is introduced by sampling "temperature deformed" determinantal point processes. The main new ingredient is a large deviation principle for Gibbs measures with singular Hamiltonians, which is proved in the present paper. As an application we show that the unique Kähler-Einstein metric with negative Ricci curvature on a canonically polarized algebraic manifold $X$ emerges in the many particle limit of the canonical point processes on $X$. In the companion paper (Berman in 2015) the extension to algebraic varieties $X$ with positive Kodaira dimension is given and a conjectural picture relating negative temperature states to the existence problem for Kähler-Einstein metrics with positive Ricci curvature is developed.
\end{abstract}

\section{Contents}

1. Introduction . . . . . . . . . . . . . . . . . . 1133

2. Submean Inequalities in Large Dimension . . . . . . . . . . . . . . . 1141

3. Proof of the Large Deviation Principle for Gibbs Measures . . . . . . . . . 1145

4. Relations to $\Gamma$-Convergence, the Gärtner-Ellis Theorem and Mean Energy 1153

5. Applications to Kähler-Einstein Geometry . . . . . . . . . . . . . . . 1158

6. Outlook . . . . . . . . . . . . . . . . . . 1167

7. Appendix: The Constant in the Cheng-Yau Gradient Estimate . . . . . . . 1169

References ....................... . . 1171

\section{Introduction}

In the present paper and the companion paper [9] a probabilistic approach to the construction of canonical metrics on a complex algebraic varieties $X$ is introduced by sam- 
pling random point processes defined in terms of algebro-geometric data, canonically attached to $X$. The processes are "positive temperature deformations" of determinantal (fermionic) point processes and the main new ingredient is a large deviation principle for Gibbs measures with singular Hamiltonians, which is proved in the present paper. As an application we show that the unique Kähler-Einstein metric with negative Ricci curvature on a canonically polarized algebraic manifold $X$ emerges in the many particle limit of the canonical point processes on $X$. More generally, in the presence of a stressenergy tensor on $X$ it is shown that the unique Kähler metric solving Einstein's equation on $X$ with negative cosmological constant (in Euclidean signature) emerges in the many particle limit.

The generalization to the construction of canonical metrics and measures on a general algebraic variety $X$ of positive Kodaira dimension are given in the companion paper [9], by exploiting the global pluripotential theory and variational calculus in $[7,12,14,20]$. This leads to a new probabilistic link between algebraic geometry on one hand (in particular the Minimal Model Program) and Kähler-Einstein geometry on the other. A conjectural picture is also developed describing the relation between the existence of negative temperature states and the existence problem for Kähler-Einstein metrics with positive Ricci curvature. In particular, relations to algebro-geometric stability properties, as in the Yau-Tian-Donaldson conjecture are described in [9]. See also [8,40] for connections to optimal transport in the real setting (corresponding to the case when $X$ is a toric and abelian variety, respectively) and [6] for connections to physics.

1.1. A large deviation principle for Gibbs measures. Let $X$ be a compact Riemannian manifold and denote by $d V$ the corresponding volume form. Given a sequence of symmetric lower semi-continuous functions $H^{(N)}$ on the $N$-fold products $X^{N}$ the corresponding Gibbs measures at inverse temperature $\beta \in] 0, \infty[$ is defined as the following sequence of symmetric probability measures on $X^{N}$ :

$$
\mu_{\beta}^{(N)}:=e^{-\beta H^{(N)}} d V^{\otimes N} / Z_{N, \beta}
$$

where the normalizing constant

$$
Z_{N, \beta}:=\int_{X^{N}} e^{-\beta H^{(N)}} d V^{\otimes N}
$$

is called the (N-particle) partition function. The ensemble $\left(X^{N}, \mu_{\beta}^{(N)}\right)$ defines a random point process with $N$ particles on $X$ which, from the point of view of statistical mechanics, models $N$ identical particles on $X$ interacting by the Hamiltonian (interaction energy) $H^{(N)}$ in thermal equilibrium at inverse temperature $\beta$. The corresponding empirical measure is the random measure

$$
\delta_{N}: X^{N} \rightarrow \mathcal{M}_{1}(X), \quad\left(x_{1}, \ldots, x_{N}\right) \mapsto \delta_{N}\left(x_{1}, \ldots, x_{N}\right):=\frac{1}{N} \sum_{i=1}^{N} \delta_{x_{i}}
$$

taking values in the space $\mathcal{M}_{1}(X)$ of all normalized positive measures on $X$, i.e., the space of all probability measures on $X$.

A classical problem is to establish conditions for the existence of a macroscopic limit of the empirical measures $\delta_{N}$ in the many particle limit $N \rightarrow \infty$. More precisely, 
the problem is to show that the random measures $\delta_{N}$ admit a deterministic limit $\mu_{\beta} \in$ $\mathcal{M}_{1}(X)$ in the sense that the law

$$
\Gamma_{N}:=\left(\delta_{N}\right)_{*} \mu_{\beta}^{(N)}
$$

of $\delta_{N}$, defining a probability measure on $\mathcal{M}_{1}(X)$, converges, as $N \rightarrow \infty$, weakly to a Dirac mass concentrated on some $\mu_{\beta}$ in $\mathcal{M}_{1}(X)$. Equivalently, the marginals $\left(\mu_{\beta}^{(N)}\right)_{j}$ of $\mu_{\beta}^{(N)}$ on $X^{j}$ satisfy

$$
\left(\mu_{\beta}^{(N)}\right)_{j}:=\int_{X^{N-j}} \mu_{\beta}^{(N)} \rightarrow \mu_{\beta}^{\otimes j},
$$

weakly as probability measures on $X^{j}$ as $N \rightarrow \infty$, which in the terminology of Kac and Snitzmann [50] means that the sequence $\mu_{\beta}^{(N)}$ is chaotic. A stronger exponential notion of convergence of $\delta_{N}$, with an explicit speed and rate functional, is offered by the theory of large deviations, by demanding that the laws $\Gamma_{N}$ satisfy a Large Deviation Principle $(L D P)$ with speed $r_{N}$ and a rate functional $F$, symbolically expressed as

$$
\Gamma_{N}(\mu) \sim e^{-r_{N} F(\mu)}, N \rightarrow \infty
$$

and assuming that $F$ admits a unique minimizer $\mu_{\beta}$ in $\mathcal{M}_{1}(X)$. Loosely speaking this means that the probability of finding a cloud of $N$ points $x_{1}, \ldots, x_{N}$ on $X$ such that the corresponding measure $\frac{1}{N} \sum_{i} \delta_{x_{i}}$ approximates a volume form $\mu$ is exponentially small unless $\mu$ is the minimizer $\mu_{\beta}$ of $F_{\beta}$.

Our main general result establishes such a LDP for a class of singular Hamiltonians:

Theorem 1.1. Let $H^{(N)}$ be a sequence of functions (Hamiltonians) on $X^{N}$ as above. Assume that

- there exists a sequence $\beta_{N} \rightarrow \infty$ of positive numbers $\beta_{N}$ such that for any continuous function $u$ on $X$

$$
\mathcal{F}_{\beta_{N}}(u):=-\frac{1}{N \beta_{N}} \log \int_{X^{N}} e^{-\beta_{N}\left(H^{(N)}\left(x_{1}, \ldots, x_{N}\right)+u\left(x_{1}\right)+\cdots+u\left(x_{N}\right)\right)} d V^{\otimes N}
$$

converges, as $N \rightarrow \infty$, to a Gateaux differentiable functional $\mathcal{F}(u)$ on $C^{0}(X)$

- $H^{(N)}$ is uniformly quasi-superharmonic, i.e. $\Delta_{x_{1}} H^{(N)}\left(x_{1}, x_{2}, \ldots x_{N}\right) \leq C$ on $X^{N}$

Then, for any fixed $\beta>0$, the measures $\left(\delta_{N}\right)_{*}\left(e^{-\beta H^{(N)}} d V^{\otimes N}\right)$ on $\mathcal{M}_{1}(X)$ satisfy, as $N \rightarrow \infty$, a large deviation principle $(L D P)$ with speed $\beta N$ and good rate functional

$$
F_{\beta}(\mu)=E(\mu)+\frac{1}{\beta} D_{d V}(\mu)
$$

where the functional $E(\mu)$ is the Legendre-Fenchel transform of $-\mathcal{F}(-\cdot)$ and $D_{d V}(\mu)$ is the entropy of $\mu$ relative to $d V$. In particular, the empirical measures $\delta_{N}$ of the corresponding random point processes on $X$ converge in law to the deterministic measure given by the unique minimizer $\mu_{\beta}$ of $F_{\beta}$. Moreover, if the equation

$$
d \mathcal{F}_{\mid u}=\frac{e^{\beta u} d V}{\int_{X} e^{\beta u} d V}
$$

on $C^{0}(X)$ admits a solution $u_{\beta}$, then the corresponding differential $\mu_{\beta}:=d \mathcal{F}_{\mid u_{\beta}}$ is the minimizer of $F_{\beta}$. 
It follows from the previous theorem that the LDP indeed also holds for the corresponding Gibbs measures with the rate functional $F_{\beta}-C_{\beta}$, where $C_{\beta}$ is the following constant:

$$
C_{\beta}:=\inf _{\mathcal{M}_{1}(X)} F_{\beta}=-\lim _{N \rightarrow \infty} \frac{1}{N \beta_{N}} \log Z_{N, \beta_{N}},
$$

It should be stressed that even the convergence of the first marginals of $\mu_{\beta}^{(N)}$, implied by the previous theorem, appears to be a new result.

As explained in Sect. 4.1 the asymptotics in the first assumption of the theorem may be replaced by the weaker assumption that there exists a functional $E(\mu)$ on $\mathcal{M}_{1}(X)$ such that

$$
H^{(N)}\left(x_{1}, \ldots, x_{N}\right) / N \rightarrow E(\mu)
$$

in the sense of Gamma convergence. Moreover, Theorem 1.1 can be viewed as a generalization of the Gärtner-Ellis theorem in the setting of Gibbs measures (see Sect. 4.2). Let us also point out that the restriction that $X$ be compact can be removed if suitable growth-assumptions of $H^{(N)}$ "at infinity" are made. But since our main application concerns the case of compact complex manifolds, we have, for simplicity, taken $X$ to be compact.

It may be illuminating to point out that in thermodynamical terms the content of Theorem 1.1 can be heuristically expressed as follows. Imagine that we know the macroscopic ground state (i.e., the state of zero energy $E$ ) of a system of a large number $N$ of particles in thermal equilibrium at zero temperature (i.e. at $\beta=\infty$ ). If we can rule out any first order phase transitions at zero-temperature (which essentially means that the macroscopic equilibrium states is unique), then increasing the temperature leads to a new macroscopic equilibrium state, minimizing the corresponding free energy functional $E-S / \beta$, where $S$ is the physical entropy (i.e., $S=-D$ with our sign conventions). In fact, in the complex geometric setting to which we next turn the zero-temperature limit $\beta \rightarrow \infty$ is reminiscent of a (second order) gas-liquid phase transition [10].

1.2. Application to Kähler-Einstein geometry. Let now $X$ be an $n$-dimensional complex algebraic projective variety of positive Kodaira dimension. This means that the plurigenera $N_{k}$ of $X$ are increasing:

$$
N_{k}:=\operatorname{dim}_{\mathbb{C}} H^{0}\left(X, k K_{X}\right) \rightarrow \infty,
$$

where $H^{0}\left(X, k K_{X}\right)$ denotes, as usual, the complex vector space of all pluricanonical (holomorphic) $n$-forms of $X$ at level $k$, i.e. $H^{0}\left(X, k K_{X}\right)$ is the space of all global holomorphic sections of the $k$ tensor power of the canonical line bundle

$$
K_{X}:=\Lambda^{n}\left(T^{*} X\right)
$$

of $X$ (using additive notation of tensor powers). In terms of local holomorphic coordinates $z_{1}, \ldots, z_{n}$ on $X$ this simply means that the elements $s^{(k)}$ of $H^{0}\left(X, k K_{X}\right)$ may be represented by local holomorphic functions $s^{(k)}$ on $X$, such that $\left|s^{(k)}\right|^{2 / k}$ transforms as a density on $X$ and thus defines a measure on $X$. To any such algebraic variety $X$ we can associate the following canonical sequence of probability measures $\mu^{\left(N_{k}\right)}$ on $X^{N_{k}}$ :

$$
\mu^{\left(N_{k}\right)}:=\frac{1}{Z_{N_{k}}}\left|\left(\operatorname{det} S^{(k)}\right)\left(z_{1}, \ldots, z_{N_{k}}\right)\right|^{2 / k},
$$


where $\operatorname{det} S^{(k)}$ is a generator of the top exterior power $\Lambda^{N_{k}}\left(H^{0}\left(X^{N_{k}}, k K_{X^{N_{k}}}\right)\right.$, i.e., totally antisymmetric (and thus defined up to a multiplicative complex number) and $Z_{N_{k}}$ is the normalizing constant. The probability measure $\mu^{\left(N_{k}\right)}$ thus defined is symmetric, i.e., invariant under the natural action of the permutation group $S_{N_{k}}$, independent of the choice of generator det $S^{(k)}$ and hence defines a canonical random point process on $X$ with $N_{k}$ points.

As shown in the companion paper [9], it follows from Theorem 1.1, combined with the asymptotics in [12] that the corresponding empirical measures $\delta_{N_{k}}$ converge in law, as $k \rightarrow \infty$, towards a deterministic measure $\mu_{c a n}$ on $X$, which is thus canonically attached to $X$. In fact, using the pluripotential theory and variational calculus in $[7,14]$ the limiting measure $\mu_{c a n}$ is shown to coincide with the canonical measure of SongTian [51] and Tsuji [52] previously defined in terms of Kähler-Einstein geometry or equivalently as solutions to certain complex Monge-Ampère equations. In the present paper we will show how to apply Theorem 1.1 in the special case when $K_{X}$ is positive (i.e. ample) to deduce the following

Theorem 1.2. Let $X$ be a compact complex manifold with positive canonical line bundle $K_{X}$. Then the empirical measures $\delta_{N_{k}}$ of the corresponding canonical random point processes on $X$ converge in law, as $N_{k} \rightarrow \infty$, towards the normalized volume form $d V_{K E}$ of the unique Kähler-Einstein metric $\omega_{K E}$ on X. More precisely, the law of $\delta_{N_{k}}$ satisfies a large deviation principle with speed $N_{k}$ whose rate functional may be identified with Mabuchi's K-energy functional on the space of Kähler metrics in $c_{1}\left(K_{X}\right)$.

By the celebrated Aubin-Yau theorem $[3,53]$ the canonical line bundle $K_{X}$ of a compact complex manifold $X$ is positive precisely when $X$ admits a Kähler-Einstein metric $\omega_{K E}$ with negative Ricci curvature, i.e., a Kähler metric with constant negative Ricci curvature:

$$
\operatorname{Ric} \omega_{K E}=-\omega_{K E}
$$

However, there are very few examples where the Kähler-Einstein metric can be obtained explicitly. The previous theorem provides a canonical sequence of quasi-explicit Kähler forms $\omega_{k}$ approximating $\omega_{K E}$ :

Corollary 1.3. Let $X$ be a complex compact manifold such that $K_{X}$ is positive. Then the sequence

$$
\omega_{k}:=d d^{c} \log \int_{X^{N_{k}-1}}\left|\left(\operatorname{det} S^{(k)}\right)\left(\cdot, x_{1}, \ldots, x_{N_{k}-1}\right)\right|^{2 / k}
$$

(consisting of Kähler forms, for $k$ sufficiently large) converges, as $k \rightarrow \infty$, to the Kähler-Einstein metric $\omega_{K} E$ in the weak topology of currents on $X$.

Theorem 1.2 fits into a more general setting of "temperature deformed" determinantal point processes attached to a polarized manifold $(X, L)$, i.e., a compact complex manifolds $X$ endowed with a positive line bundle $L$ (Theorem 5.7). More precisely, in the general setting the point processes are attached to the data $\left(\|\cdot\|, d V, \beta_{k}\right)$ consisting of a Hermitian metric $\|\cdot\|$ on a $L$, a volume form $d V$ on $X$ and a sequence of positive numbers $\left.\left.\beta_{k} \rightarrow \beta \in\right] 0, \infty\right]$. Then the corresponding probability measures on $X^{N_{k}}$ are defined by

$$
\mu^{\left(N_{k}, \beta\right)}:=\frac{\left\|\left(\operatorname{det} S^{(k)}\right)\left(x_{1}, x_{2}, \ldots x_{N_{k}}\right)\right\|^{2 \beta_{k} / k} d V^{\otimes N_{k}}}{Z_{N_{k}, \beta}},
$$


where $\operatorname{det} S^{(k)}$ is a generator of the top exterior power $\Lambda^{N_{k}} H^{0}(X, k L)$. Concretely, the corresponding LDP is equivalent to the following asymptotics for the $L^{2 \beta_{k} / k}$-norm of the generator det $S^{(k)}$ of the determinant line of $H^{0}(X, k L)$ which is orthonormal with respect to the $L^{2}$-product determined by $(\|\cdot\|, d V)$ :

$$
\frac{1}{N_{k}} \log \left\|\operatorname{det} S^{(k)}\right\|_{L^{2 \beta_{k} / k}\left(X^{\left.N_{k}, \mu_{0}^{\otimes N_{k}}\right)}\right.} \rightarrow-\inf _{\mu \in \mathcal{M}_{1}(X)} F_{\beta}(\mu)
$$

(by Lemma 4.7). In this general setting the limiting deterministic measure $\mu_{\beta}$ minimizing $F_{\beta}$ is the volume form of the unique Kähler metric $\omega_{\beta}$ in the first Chern class of $L$ solving the twisted Kähler-Einstein equation

$$
\operatorname{Ric} \omega=-\beta \omega+\eta,
$$

where the twisting form $\eta$ is explicitly determined by $(\|\cdot\|, d V, \beta)$. The point is that when $L=K_{X}$ any given volume form $d V$ naturally defines a metric $\|\cdot\|_{d V}$ on $L$ and the probability measures on $X^{N_{k}}$ attached to $\left(\|\cdot\|_{d V}, d V, 1\right)$ are precisely the canonical ones defined by formula 1.6. Moreover, in this special case $\eta$ vanishes and the Eq. 1.10 thus reduces to the usual Kähler-Einstein equation 1.7. The more general twisted version of the equation has previously appeared in various situations in Kähler geometry [37, $51,52]$. From the physics point of view the twisting form $\eta$ corresponds to the (tracereversed) stress-energy tensor in Einstein's equations on $X$ (with Euclidean signature).

The Hamiltonians

$$
H^{\left(N_{k}\right)}\left(x_{1}, \ldots, x_{N_{k}}\right):=-k^{-1} \log \left\|\left(\operatorname{det} S^{(k)}\right)\left(x_{1}, x_{2}, \ldots x_{N_{k}}\right)\right\|^{2}
$$

corresponding to the probability measures 1.9 are strongly non-linear unless $X$ is a Riemann surface, i.e. unless $n=1$. In fact, in the simplest latter case, i.e., when $X$ is the Riemann sphere, $H^{\left(N_{k}\right)}\left(x_{1}, \ldots, x_{N_{k}}\right)$ is a sum of identical pair interactions $W\left(x_{i}, x_{j}\right)$, where $W$ is the Green function of the corresponding Laplace operator and then the corresponding functional $E(\mu)$ is the Dirichlet energy (Remark 5.11). In general, the connection to the Kähler-Einstein geometry of $(X, L)$ will be shown to arise from the fact that the Eq. 1.4 is intimately related to the complex Monge-nAmpère equation

$$
\left(\omega_{0}+i \partial \bar{\partial} u\right)^{n}=e^{\beta u} d V,
$$

where $\omega_{0}$ is the normalized curvature two form of the given metric $\|\cdot\|$ on $L$. More precisely, the two equations coincide for smooth functions $u$ such that $\omega_{0}+i \partial \bar{\partial} u$ is a Kähler form (i.e., smooth and positive). In this complex geometric setting the strong non-linearity of the Hamiltonians $H^{(N)}$ when $n \geq 2$ is reflected in the non-linearity of the complex Monge-Ampère operator appearing in the left hand side of Eq. 1.12 (coinciding with the Laplacian when $n=1$ ). Furthermore, the singularity of $H^{(N)}$ (which is present for any dimension $n$ ) is a reflection of the fact that solutions to the (generalized) Calabi-Yau equation

$$
\left(\omega_{0}+i \partial \bar{\partial} u\right)^{n}=\mu
$$

are, in general, singular when $\mu$ is a probability measure on $X$ (as is clear already for the Laplace equation appearing when $n=1$ ).

Finally, let us point out that the extension to general complex algebraic manifolds $X$ with positive Kodaira dimension, established in the companion paper [9], relies on an extension of Theorem 5.7 to line bundles $L$ which are big (but not necessarily positive); see Sect. 5.4. 
1.3. Comparison with previous results. First a comment on relations to the physics literature: in the case $n=1$ (i.e., in two real dimensions) the quasi-linear Laplace type Eq. 1.12 arises as the macroscopic equilibrium equation in a range of statistical mechanical models of mean field type: it is called the Joyce-Montgomery equation in Onsager's vortex model for 2D turbulence, the Poisson-Boltzmann equation in the Debye-Hückel theory of plasmas and electrolytes and the Lane-Emden equation in stellar physics (see [35]). But the Monge-Ampère equation $(n>1)$ does not seem to have appeared in any statistical mechanical model before. On the other hand, in the case when $\beta_{k}:=k$ the density of the corresponding probability measure has a natural quantum mechanical interpretation: it is the squared amplitude of the Slater determinant representing a maximally filled many particle state of $N$ free fermions on $X$, subject to an exterior magnetic field (the corresponding single particle wave functions are elements of $H^{0}(X, k L)$ and represent the corresponding lowest Landau levels). The case when $\beta_{k}=\nu k$, for a given positive integer $v$, also appears in the fractional Quantum Hall Effect, where the corresponding probability density is the squared amplitude of the Laughlin state (see the review [43] and references therein).

1.3.1. Large deviations. The LDP in Theorem 1.1 in the case when $H^{(N)}$ is uniformly equicontinuous is essentially well-known in the setting of mean field models [8,34] (it then also applies to the case of negative $\beta$, by replacing $H^{(N)}$ with $\left.-H^{(N)}\right)$. But the key feature of Theorem 1.1 is that it applies to a large class of singular Hamiltonians and in particular $H^{(N)}$ is allowed to be strongly repulsive in the sense that it blows up, as two points merge (and hence the Gibbs measure may be ill-defined when $\beta$ is negative). It seems that the only previous class where a convergence result as in Theorem 4.6 has been established for singular Hamiltonians is in the "linear" case when $H^{(N)}$ is a sum of pair interactions with a mean field scaling:

$$
H^{(N)}\left(x_{1}, \ldots, x_{N}\right)=\frac{1}{(N-1)} \sum_{1 \leq i<j \leq N} W\left(x_{i}, x_{j}\right),
$$

where the pair interaction $W$ is allowed to be singular along the diagonal, as long it is lower semi-continuous and in $L_{\text {loc }}^{1}$ (this is indeed a mean field interaction in the sense that each particle $x_{i}$ is exposed to the average of the pair interactions $W\left(x_{i}, x_{j}\right)$ for the $N-1$ remaining particles). Then the asymptotics of the partitions functions 1.5 can be obtained using the method of Messer-Spohn [45], which is based on the Gibbs variational principle and which crucially relies on the existence of the mean energy $\bar{E}(\mu)$ corresponding to $H^{(N)}$ (see $[26,41]$ for the case of a logarithmic singularity which is motivated by Onsager's vortex model for 2D turbulence $[35,46])$. A similar argument applies in the case of "finite order", i.e., when $H^{(N)}$ is a sum of $j$-point interactions for a uniformly bounded $j$ (then $E(\mu)$ depends polynomially on $\mu$ ). However, the main point of the previous theorem is to avoid the latter assumption, which is not satisfied in the application to Kähler-Einstein geometry (apart from the classical lowest dimensional setting of Riemann surfaces). In particular, the present proof bypasses the problem of the existence of the limiting mean energies. Instead, the main idea of the proof is to exploit the Riemannian orbifold geometry of the space of configurations of $N$ points on $X$, viewed as the singular quotients $X^{N} / S_{N}$, where $S_{N}$ is the symmetric group acting on $X^{N}$ by permuting the factors. The key result is a submean inequality for positive quasisubharmonic functions on $X^{N} / S_{N}$ with a distortion coefficient that is sub-exponential in the dimension (Theorem 2.1), which is closely related to an inequality of Li-Schoen [44]. 
There is also another approach to large deviation principles for mean field Hamiltonians of the form 1.14 originating in the literature on random matrices and Coulomb gases $[17,18,28,48]$, which as explained in [48], is closely related to the notion of Gamma convergence (see also $[55,56]$ for applications to univariat random polynomials). This approach seems to be limited to the case when $\beta_{N} \gg \log N$ and in particular $\beta=\infty$ so that the entropy contributions can be neglected. ${ }^{1}$ See also [33] for a general LDP for Hamiltonians of the form 1.14 using weak convergence methods.

Let us also point out that the role of $\left(\operatorname{det} S^{(k)}\right)\left(x_{1}, x_{2}, \ldots x_{N_{k}}\right)$ appearing in formula 1.9 is played by the classical Vandermonde determinant in the random matrix literature (see Example 5.6). In fact, there is a non-compact analogue of Theorem 5.7 in Euclidean $\mathbb{C}^{n}$ which specializes to the setting of random matrix theory and the $2 \mathrm{D}$ log gas when $n=1$ and $\beta=\infty$ and to the 2D vortex model (for $n=1$ and $\beta<\infty$ ) and which can be proved by supplementing the proof of Theorem 5.7 with a tightness estimate, as in the non-compact setting considered for $\beta=\infty$ in [5] (see also [19] for the case $\beta=\infty$ ). Details will appear elsewhere.

1.3.2. Kähler geometry. A statistical mechanics approach has previously been applied to conformal geometry [42], as opposed to the present complex-geometric setting. The role of the "determinantal" Hamiltonian 1.11 is in the conformal setting played by a mean field Hamiltonian of the form 1.14 with a logarithmic pair interaction and the role of the fully non-linear complex Monge-Ampère operator is played by a linear conformally invariant operator, which is zero-order perturbation of a power of the Laplacian (the Paneitz operator). Accordingly, previous results in $[26,41]$ concerning such Hamiltonians can be applied in the conformal setting (compare the discussion above), while the present setting seems to require new methods.

The present probabilistic should be viewed in the light of the pervasive philosophy in Kähler geometry, going back to Yau [54], of approximating metrics on a complex algebraic manifold with algebraically defined Bergman metrics, which may be identified with elements of the symmetric space $G L(N, \mathbb{C}) / U(N)$. For example, the quasi-explicit Kähler metrics $\omega_{k}$ in formula 1.8, approximating the Kähler-Einstein metric $\omega_{K E}$ on a canonically polarized manifold $X$, are analogs of Donaldson's balanced metrics in $G L(N, \mathbb{C}) / U(N)$ [32]. One advantage of the present approach is that, as shown in the companion paper [9], the approximation also applies when $X$ is of general type, where the role of $\omega_{K E}$ is played by the canonical Kähler-Einstein current on $X$ (which is singular along a subvariety of $X$ ) [14,20]. In another direction it would be interesting to see if the present approach can be implemented to construct numerical simulations of Kähler-Einstein metrics, using Monte Carlo type methods, complementing the different numerical approaches in [31,32] (see [4] for relations between Monte Carlo simulations and similar polynomial determinantal point processes).

Even if the connection between canonical random point processes on a complex algebraic manifold $X$ does not seem to have been studied before, there are some connections to previous work on random polynomials/holomorphic sections in a given back-ground geometry [49]; in particular in the one-dimensional setting where an LDP was obtained in $[55,56]$. Another probabilistic approach to the space of Kähler metrics has been introduced in a series of papers by Ferrari, Klevtsov and Zelditch [36], motivated by Quantum Field Theory. The approach aims at approximating random Kähler metrics with random Bergman metrics. Accordingly, the role of the $N$-particle space $X^{N} / S_{N}$ is in [36] played

\footnotetext{
1 The Hamiltonians in the random matrix and Coulomb gas literature are usually scaled in a different way so that our zero-temperature $(\beta=\infty)$ corresponds to a fixed inverse temperature.
} 
by the symmetric space $G L(N . \mathbb{C}) / U(N)$. In conclusion, it would be very interesting to understand the precise connections between [36] and the present setting, as well as the connection to Donaldson's balanced metrics [32].

Organization. In Sect. 2, we prove the submean inequality in large dimensions, which plays a key role in the subsequent Sect. 3, where the general LDP in Theorem 1.1 is proved. In Sect. 4, we make a digression on relations to previous methods and notions used in the literature on large deviations. The applications to Kähler-Einstein geometry are given in Sect. 5. For the convenience of readers lacking background in Kähler geometry we start the section by giving a reasonably self-contained account of the Kähler geometry setup (including some rudiments of pluripotential theory). The article is concluded with an outlook in Sect. 6 on some open problems and an appendix where the dimension dependence on the constant in the Cheng-Yau gradient estimate is obtained by tracing through the usual proof.

\section{Submean Inequalities in Large Dimension}

2.1. Setup. Let $(X, g)$ be a $n$-dimensional Riemannian manifold and assume that

$$
\text { Ric } g \geq-\kappa^{2}(n-1) g
$$

for some positive constant $\kappa$ (sometimes referred to as the normalized lower bound on the Ricci curvature). Let $G$ a finite group acting by isometries on $X$ and denote by $M:=X / G$ the corresponding quotient equipped with the distance function induced by the metric $g$, i.e.

$$
d_{M}(x, y):=\inf _{\gamma \in G} d_{X}(x, \gamma y)
$$

where $d_{X}$ is the Riemannian distance function on $(X, g)$. Even though the quotient $M$ is not a manifold in general (since $G$ will in general have fixed points) it still comes with a smooth structure in the following sense. Denote by $p$ the natural projection map from $X$ to $M$. Using the projection $p$ we can identify a function $f$ on $M$ with $G$-invariant function $p^{*} f$ on $X$ and accordingly we say that $f$ is smooth if $p^{*} f$ is. Similarly, there is a natural notion of Laplacian $\Delta$ on the quotient $M$ : the Laplacian $\Delta u$ of a locally integrable function $u$ on $M$ is the signed Radon measure defined by

$$
\int_{M}(\Delta u) f:=\frac{1}{|G|} \int_{X} p^{*} u \Delta\left(p^{*} f\right)
$$

for any smooth function $f$ on $M$. More generally, by localization, this setup naturally extends to the setting of Riemannian orbifolds (see [21]), but the present setting of global quotients will be adequate for our purposes.

\subsection{Statement of the submean inequality.}

Theorem 2.1. Let $(X, g)$ be a Riemannian manifold of dimension $n$ such that Ric $g \geq-\kappa^{2}(n-1) g$ and $G$ a finite group acting by isometries on $X$. Denote by $M:=X / G$ the corresponding quotient equipped with the distance function induced by the metric $g$ and let $v$ be a non-negative function on $M$ such that $\Delta_{g} v \geq-\lambda^{2} v$ for 
some non-negative constant $\lambda$. Then, for any $\delta \in] 0,1[$ and $\epsilon \in] 0,1]$ there exist constants $A$ and $C$ such that

$$
\sup _{B_{\epsilon \delta}\left(x_{0}\right)} v^{2} \leq A e^{2 \lambda \delta} e^{C n(\delta+\epsilon)} \frac{\int_{B_{\delta}\left(x_{0}\right)} v^{2} d V}{\int_{B_{\epsilon \delta}\left(x_{0}\right)} d V},
$$

where $C$ only depends on an upper bound on $\kappa$ and A only depends on $\delta$ and $\epsilon$ (assuming that the balls above are contained in a compact subset of $M$ ).

Note that by the $G$-invariance we may as well replace the functional $v$ and the balls on $M$ with their pull-back to $X$.

2.3. Proof of the submean inequality in Theorem 2.1. We will follow closely the elegant proof of Li-Schoen [44] of a similar submean inequality. But there are two new features here that we have to deal with:

- We have to make explicit the dependence on the dimension $n$ of all constants and make sure that the final contribution is sub-exponential in $n$

- We have to adapt the results to the singular setting of a Riemannian quotient

Before turning to the proof we point out that it is well-known that submean inequalities with a multiplicative constant $C(n)$ do hold in the more general singular setting of Alexandrov spaces (with a strict lower bound $-\kappa$ on the sectional curvature). But it seems that the current proofs (see for example [38]), which combine local Poincaré and Sobolev inequalities with the Moser iteration technique, do not give the subexponential dependence on $C(n)$ that we need.

We recall that the two main ingredients in the proof of the result of Li-Schoen referred to above is the gradient estimate of Cheng-Yau [29] and a Poincaré-Dirichlet inequality on balls. Let us start with the gradient estimate that we will need:

Proposition 2.2. Let $u$ be a harmonic function on the ball $B_{a}\left(x_{0}\right)$ of radius a centered at $x_{0} \in M$ and assume that $a \leq 1$ Set $\rho_{x_{0}}(x):=d\left(x, x_{0}\right)$ (the distance between $x$ and $\left.x_{0}\right)$. Then

$$
\sup _{B_{a}\left(x_{0}\right)}\left(|\nabla \log u|\left(a-\rho_{x_{0}}\right)\right) \leq C n,
$$

where the constant $C$ only depends on an upper bound on $\kappa$.

Proof. In the smooth case this is the celebrated Cheng-Yau gradient estimate [29]. The result is usually stated without an explicit estimate of the multiplicative constant $C_{n}$ in terms of $n$, but tracing through the proof in [29] gives $C_{n} \leq C n$ (see the appendix in the present paper and also [2] for a probabilistic proof providing an explicit constant). We claim that the same estimate holds in the present setting using a lifting argument. To see this recall that the usual proof of the gradient estimate proceeds as follows (see the appendix). Set $\phi(x):=|\nabla \log u|(=|\nabla u| / u)$ and $F(x):=\phi(x)\left(\rho_{x_{0}}-a\right)^{2}$. Then $F$ attains its maximum in a point $x_{1}$ in the interior of $B_{a}\left(x_{0}\right)$ (otherwise $|\nabla u|$ vanishes identically and then we are trivially done). Hence, $F(x) \leq F\left(x_{1}\right)$ on some neighborhood $U$ of $x_{1}$. Now, in case $F$ (or equivalently $\rho_{x_{0}}$ ) is smooth on $U$ we get $\Delta F \leq 0$ and $\nabla F=0$ at $x_{1}$. Calculating $\Delta F$ and using Bochner formula and Laplacian comparison then gives

$$
\phi\left(x_{1}\right)\left(a-\rho_{x_{0}}\left(x_{1}\right)\right) \leq C n
$$


which is the desired estimate. In the case when $\rho_{x_{0}}$ is not smooth on $U$, i.e. $x_{1}$ is contained in the cut locus of $x_{0}$ one first replaces $\rho_{x_{0}}$ with a smooth approximation $\rho_{x_{0}}^{(\epsilon)}$ of $\rho_{x_{0}}$ (which is a local barrier for $\rho_{x_{0}}$ ) and then lets $\epsilon \rightarrow 0$ to get the same conclusion as before. In the singular case $M=X / G$ we proceed as follows. First we identify $F$ with a $G$-invariant function on the inverse image of $B_{R}\left(x_{0}\right)$ in $X$ (and $x_{0}$ and $x_{1}$ with a choice of lifts in the corresponding $G$-orbits) and set $\tilde{F}:=\phi(x)\left(a-\tilde{\rho}_{x_{0}}\right)^{2}$, where $\tilde{\rho}_{x_{0}}(x):=d_{X}\left(x_{0}, x\right)$. By definition $\tilde{\rho}_{x_{0}} \geq \rho_{x_{0}}$ on $X$ and, after possibly changing the lift of the point $x_{1}$ we may assume that $\tilde{\rho}_{x_{0}}=\rho_{x_{0}}$ at $x=x_{1}$ and hence $\tilde{\rho}_{x_{0}}<a$ (after perhaps shrinking $U$ ). In particular, $\tilde{F} \leq F$ on $U$ and $\tilde{F}=F$ at $x_{1}$ and hence $\tilde{F}$ also has a local maximum at $x_{1}$. But then the previous argument in the smooth case gives that 2.1 holds with $\rho_{x_{0}}$ replaced by $\tilde{\rho}_{x_{0}}$. But since the two functions agree at $x_{1}$ this concludes the proof in the general case.

Corollary 2.3. Let $h$ be a positive harmonic function on $B_{\delta}\left(x_{0}\right)$. Then there exists a constant $C$ only depending on an upper bound on $\kappa$ such that

$$
\sup _{B_{\epsilon \delta}\left(x_{0}\right)} h^{2} \leq e^{C \epsilon n} \frac{\int_{B_{\epsilon \delta}\left(x_{0}\right)} h^{2} d V}{\int B_{\epsilon \delta}\left(x_{0}\right) d V}
$$

for $0<\epsilon<1$.

Proof. Set $v:=\log h$ and fix $x \in B_{\epsilon \delta}\left(x_{0}\right)$. Integrating along a minimizing geodesic connecting $x_{0}$ and $x$ and using the gradient estimate in the previous proposition gives

$$
\left|v(x)-v\left(x_{0}\right)\right| \leq C n \int_{0}^{\epsilon \delta} \frac{1}{\delta-t} d t=C n(\log (\delta-0)-\log (\delta-\epsilon \delta))=-C n \log (1-\epsilon) \text {. }
$$

In particular, for any two points $x, y \in B_{\epsilon \delta}\left(x_{0}\right)$ we get $|v(x)-v(y)| \leq\left|v(x)-v\left(x_{0}\right)\right|+$ $\left|v(y)-v\left(x_{0}\right)\right| \leq-2 C n \log (1-\epsilon)$, i.e. $h(x) \leq(1-\epsilon)^{-2 C n} h(y)$. In particular, $\sup _{B_{\epsilon \delta}\left(x_{0}\right)} h^{2} \leq(1-\epsilon)^{-4 C n} \inf _{B_{\epsilon \delta}\left(x_{0}\right)} h^{2}$, which implies the proposition after renaming the constant $C$.

The second key ingredient in the proof of Theorem 2.1 is the following Poincaré-Dirichlet inequality:

Proposition. Let $f$ be a smooth function on $B_{\delta}\left(x_{0}\right)$ vanishing on the boundary. Then

$$
\int_{B_{\delta\left(x_{0}\right)}}|f|^{2} d V_{g} \leq 4 e^{C n \delta} \int_{B_{\delta\left(x_{0}\right)}}|\nabla f|^{2} d V_{g}
$$

where the constant $C$ only depends on an upper bound on $\kappa$.

Proof. We follow the proof in [44] with one crucial modification (compare the remark below). To fix ideas we first consider the case of a Riemannian manifold. Fix a point $p$ in the boundary of the ball $B_{1}\left(x_{0}\right)$ and denote by $r_{1}(x)$ the distance between $x \in M$ and $p$. From the standard comparison estimate for the Laplacian we get

$$
\Delta r_{1} \leq(n-1)\left(\frac{1}{r_{1}}+\kappa\right)
$$


(in the weak sense and point-wise away from the cut locus of $p$ ). In particular, for any positive number $a$ we deduce the following inequality on $B_{\delta}\left(x_{0}\right)$ (using that $g\left(\nabla r_{1}, \nabla r_{1}\right)=$ 1) a.e.)

$$
\Delta_{g}\left(e^{-a r_{1}}\right)=a e^{-a r_{1}}\left(a-\Delta r_{1}\right) \geq a e^{-a(1+\delta)}\left(a-(n-1)\left(\frac{1}{(1-\delta)}+\kappa\right)\right)
$$

Hence, setting $a:=n\left(\frac{1}{(1-\delta)}+\kappa\right)$ gives

$$
\Delta_{g}\left(e^{-a r_{1}}\right) \geq a e^{-a(1+\delta)}\left(\frac{1}{(1-\delta)}+\kappa\right)>0
$$

Multiplying by $|f|$ and integrating once by parts (and using that $\left\|\nabla r_{1}\right\| \leq 1$ ) we deduce that

$$
a \int_{B_{\delta\left(x_{0}\right)}}|\nabla f| e^{-a r_{1}} d V \geq a\left(\frac{1}{(1-\delta)}+\kappa\right) \int_{B_{\delta\left(x_{0}\right)}}|f| e^{-a(1+\delta)} d V .
$$

Estimating $e^{-a r_{1}} \leq e^{-a(1-\delta)}$ in the left hand side above and rearranging gives

$$
\int_{B_{\delta\left(x_{0}\right)}}|\nabla f| d V e^{2 a \delta}\left(\frac{1}{(1-\delta)}+\kappa\right)^{-1} \geq \int_{B_{\delta\left(x_{0}\right)}}|f| d V,
$$

(using that $g\left(\nabla r_{1}, \nabla r_{1}\right) \leq 1$ in the sense of upper gradients). This shows that the $L^{1}$-version of the Poincaré inequality in question holds with the constant $\frac{1}{(1-\delta)}+$ $\kappa)^{-1} e^{\delta 2 n\left(\frac{1}{(1-\delta)}+\kappa\right)}$, which for $\delta$ sufficiently small is bounded from above by $e^{n(4+2 \kappa) \delta}$. The general Riemannian $L^{2}$-Poincare inequality now follows from replacing $|f|$ with $|f|^{2}$ and using Hölder's inequality. Finally, in the case of the a Riemannian quotient $M$ we can proceed exactly as above using that the Laplacian comparison estimate in formula 2.2 is still valid. Indeed, the pull-back $p^{*} r_{1}$ of $r_{1}$ to $X$ is an infimum of functions for which the corresponding estimate holds (by the usual Laplacian comparison estimate and the assumption that $G$ acts by isometries). But then the estimate also holds for the function $p^{*} r_{1}$, by basic properties of Laplacians. More generally, the required Laplacian comparison estimate was shown in [21] for general Riemannian orbifolds.

Remark 2.4. The only difference from the argument used in [44] is that we have taken the point $p$ to be of distance 1 from $x_{0}$ rather than distance $2 \delta$, as used in [44]. For $\delta$ small this change has the effect of improving the exponential factor from $e^{n(1+\delta \kappa)}$ to $e^{n(\delta+\delta \kappa)}$, which is crucial as we need a constant in the Poincare inequality which has subexponential growth in $n$ as $\delta \rightarrow 0$.

2.3.1. End of proof of Theorem 2.1. Let us first consider the case when $\lambda=0$. Denote by $h$ the harmonic function on $B_{\delta}$ coinciding with $v$ on $\partial B_{\delta}$. By Cor 2.3 and the subharmonicity of $v$

$$
\sup _{B_{\epsilon \delta}\left(x_{0}\right)} v^{2} \leq e^{C n \epsilon} \frac{\int_{B_{\epsilon \delta}\left(x_{0}\right)}|h|^{2} d V_{g}}{\int_{B_{\epsilon \delta}\left(x_{0}\right)} d V_{g}} .
$$

Next, by the triangle inequality

$$
\int_{B_{\epsilon \delta}\left(x_{0}\right)}|h|^{2} d V_{g} / 2 \leq \int_{B_{\epsilon \delta}\left(x_{0}\right)}|h-v|^{2} d V+\int_{B_{\epsilon \delta}\left(x_{0}\right)}|v|^{2} d V
$$


Since $h-v$ vanishes on the boundary of $B_{\delta}\left(x_{0}\right)$ applying the Poincare inequality in Prop 2.3 then gives $\int_{B_{\epsilon \delta}\left(x_{0}\right)}|h-v|^{2} d V \leq$

$$
\leq \int_{B_{\delta\left(x_{0}\right)}}|h-v|^{2} d V \leq A e^{B n \delta} \int_{B_{\delta\left(x_{0}\right)}}|\nabla h-\nabla v|^{2} d V \leq 2 A e^{B n \delta} \int_{B_{\delta\left(x_{0}\right)}}|\nabla h|^{2}+|\nabla v|^{2} d V
$$

But $h$ is the solution to a Dirichlet problem and as such minimizes the Dirichlet norm $\int_{B_{\delta\left(x_{0}\right)}}|\nabla h|^{2}$ over all subharmonic functions with the same boundary values as $h$. Accordingly,

$$
\int_{B_{\epsilon \delta}\left(x_{0}\right)}|h-v|^{2} d V \leq 4 A e^{B n \delta} \int_{B_{\delta\left(x_{0}\right)}}|\nabla v|^{2} d V
$$

Finally, using that $v$ is subharmonic we get

$$
\int_{B_{\delta\left(x_{0}\right)}}|\nabla v|^{2} d V \leq C_{\delta} \int_{B_{2 \delta\left(x_{0}\right)}}|v|^{2} d V
$$

(as is seen by multiplying with a suitable smooth function $\chi$ supported on $B_{2 \delta}$ such that $\chi=1$ on $B_{\delta}$ ). All in all this concludes the proof of Theorem 2.1 in the case $\lambda=0$.

Finally, to handle the general case (i.e. $\lambda \neq 0$ ) we set $N:=M \times]-1,1$ [ equipped with the standard product metric and apply the previous case to the function $v e^{\lambda t}$ to get

$$
\sup _{B_{\epsilon \delta}\left(x_{0}, 0\right) \subset N} v^{2} e^{2 \lambda t} \leq A_{\delta} e^{B n(\delta+\epsilon)} \frac{\int_{B_{2 \delta}\left(x_{0}, 0\right) \subset N} v^{2} e^{2 \lambda t} d V}{\int_{B_{\epsilon \delta}\left(x_{0}, 0\right) \subset N} d V},
$$

But restricting the sup in the left hand side to $B_{\epsilon \delta}\left(x_{0}\right) \times\{0\}$ and using that $B_{\epsilon \delta / 2}\left(x_{0}, 0\right) \times$ $[-\epsilon \delta / 2, \epsilon \delta / 2] \subset B_{\epsilon \delta}\left(x_{0}, 0\right)$ and $B_{2 \delta}\left(x_{0}, 0\right) \subset B_{2 \delta}\left(x_{0}, 0\right) \times[2 \delta, 2 \delta]$ gives

$$
\sup _{B_{\epsilon \delta}\left(x_{0}\right) \subset M} v^{2} \leq A_{\delta, \epsilon} e^{2 \lambda \delta} e^{B n(\delta+\epsilon)} \frac{\int_{B_{2 \delta}\left(x_{0}\right) \subset M} v^{2} d V_{g}}{\int_{B_{\epsilon \delta / 2}\left(x_{0}, 0\right) \subset M} d V_{g}},
$$

which concludes the proof of the general case (after a suitable rescaling).

\section{Proof of the Large Deviation Principle for Gibbs Measures}

Given a compact topological space $X$ we will denote by $C^{0}(X)$ the space of all continuous functions $u$ on $X$, equipped with the sup-norm and by $\mathcal{M}(X)$ the space of all signed (Borel) measures on $X$. The subset of $\mathcal{M}(X)$ consisting of all probability measures will be denoted by $\mathcal{M}_{1}(X)$. We endow $\mathcal{M}(X)$ with the weak topology, i.e. $\mu_{j}$ is said to converge to $\mu$ weakly in $\mathcal{M}(X)$ if

$$
\left\langle\mu_{j}, u_{j}\right\rangle \rightarrow\langle\mu, u\rangle
$$

for any continuous function $u$ on $X$, i.e. for any $u \in C^{0}(X)$, where $\langle u, \mu\rangle$ denotes the standard integration pairing between $C^{0}(X)$ and $\mathcal{M}(X)$ (equivalently, the weak topology is precisely the weak*-topology when $\mathcal{M}(X)$ is identified with the topological dual of $\left.C^{0}(X)\right)$. A functional $\mathcal{F}$ on $C^{0}(X)$ will be said to be Gateaux differentiable if it is differentiable along affine lines and for any $u$ in $C^{0}(X)$ there exists an element $d \mathcal{F}_{\mid u}$ in $\mathcal{M}(X)$, called the differential of $\mathcal{F}$ at $u$, such that for any $v$ in $C^{0}(X)$

$$
\frac{d \mathcal{F}(u+t v)}{d t}_{\mid t=0}=\left\langle d \mathcal{F}_{\mid u}, v\right\rangle
$$


3.1. Setup: the Gibbs measure $\mu_{\beta}^{(N)}$ associated to the Hamiltonian $H^{(N)}$. A random point process with $N$ particles is by definition a probability measure $\mu^{(N)}$ on the $N$ particle space $X^{N}$ which is symmetric, i.e. invariant under permutations of the factors of $X^{N}$. The empirical measure of a given random point process is the following random measure

$$
\delta_{N}: X^{N} \rightarrow \mathcal{M}_{1}(X), \mapsto\left(x_{1}, \ldots, x_{N}\right) \mapsto \delta_{N}\left(x_{1}, \ldots, x_{N}\right):=\frac{1}{N} \sum_{i=1}^{N} \delta_{x_{i}}
$$

on the ensemble $\left(X^{N}, \mu^{(N)}\right)$. By definition the law of $\delta_{N}$ is the push-forward of $\mu^{(N)}$ to $\mathcal{M}_{1}(X)$ under the map $\delta_{N}$, which thus defines a probability measure on $\mathcal{M}_{1}(X)$.

Now fix a back-ground measure $\mu_{0}$ on $X$ and let $H^{(N)}$ be a given $N$-particle Hamiltonian, i.e. a symmetric function on $X^{N}$, which we will assume is lower semi-continuous (and in particular bounded from below, since $X$ is assumed compact). Also fixing a positive number $\beta$ the corresponding Gibbs measure (at inverse temperature $\beta$ ) is the symmetric probability measure on $X^{N}$ defined as

$$
\mu_{\beta}^{N}:=e^{-\beta H^{(N)}} \mu_{0}^{\otimes N} / Z_{N}
$$

where the normalizing constant

$$
Z_{N, \beta}:=\int_{X^{N}} e^{-\beta H^{(N)}} \mu_{0}^{\otimes N}
$$

is called the ( $N$-particle) partition function. In our setting we will take $\mu_{0}$ to be the volume form $d V$ of a fixed Riemannian metric. Given a continuous function $u$ on $X$ we will also write

$$
Z_{N, \beta}[u]:=\int_{X^{N}} e^{-\beta\left(H^{(N)}+u\right)} \mu_{0}^{\otimes N},
$$

where $u$ has been identified with the following function on the product $X^{N}$ :

$$
u\left(x_{1}, . ., x_{N}\right):=\sum_{i=1}^{N} u\left(x_{i}\right)
$$

3.2. Preliminaries on large deviation principles and Legendre transforms. Let us start by recalling the general definition of a Large Deviation Principle (LDP) for a sequence of measures.

Definition 3.1. Let $\mathcal{P}$ be a Polish space, i.e. a complete separable metric space.

(i) A function $I: \mathcal{P} \rightarrow]-\infty, \infty]$ is a rate function if it is lower semi-continuous. It is a good rate function if it is also proper.

(ii) A sequence $\Gamma_{k}$ of measures on $\mathcal{P}$ satisfies a large deviation principle with speed $r_{k}$ and rate function $I$ if

$$
\limsup _{k \rightarrow \infty} \frac{1}{r_{k}} \log \Gamma_{k}(\mathcal{F}) \leq-\inf _{\mu \in \mathcal{F}} I
$$


for any closed subset $\mathcal{F}$ of $\mathcal{P}$ and

$$
\liminf _{k \rightarrow \infty} \frac{1}{r_{k}} \log \Gamma_{k}(\mathcal{G}) \geq-\inf _{\mu \in G} I(\mu)
$$

for any open subset $\mathcal{G}$ of $\mathcal{P}$.

Remark 3.2. The LDP is said to be weak if the upper bound is only assumed to hold when $\mathcal{F}$ is compact. Anyway, we will be mainly interested in the case when $\mathcal{P}$ is compact and hence the notion of a weak LDP and an LDP then coincide (and moreover any rate functional is automatically good).

We will be mainly interested in the case when $\Gamma_{k}$ is a probability measure (which implies that $I \geq 0$ with infimum equal to 0 ). Then it will be convenient to use the following alternative formulation of a LDP (see Theorems 4.1.11 and 4.1.18 in [30]):

Proposition 3.3. $\mathcal{P}$ be a metric space and denote by $B_{\epsilon}(\mu)$ the ball of radius $\epsilon$ centered at $\mu \in \mathcal{P}$. Then a sequence $\Gamma_{N}$ of probability measures on $\mathcal{P}$ satisfies a weak LDP with speed $r_{N}$ and a rate functional I iff

$$
\lim _{\epsilon \rightarrow 0} \liminf _{N \rightarrow \infty} \frac{1}{r_{N}} \log \Gamma_{N}\left(B_{\epsilon}(\mu)\right)=-I(\mu)=\lim _{\epsilon \rightarrow 0} \limsup _{N \rightarrow \infty} \frac{1}{r_{N}} \log \Gamma_{N}\left(B_{\epsilon}(\mu)\right)
$$

We note the following simple lemma which allows one to extend the previous proposition to the non-normalized measures $\left(\delta_{N}\right)_{*} e^{-\beta H^{(N)}} \mu_{0}^{\otimes N}$ :

Lemma 3.4. Assume that the following bound for the partition functions holds: $\left|\log Z_{N, \beta}\right| \leq C N$. Then the measures

$$
\Gamma_{N}:=\left(\delta_{N}\right)_{*} e^{-\beta H^{(N)}} \mu_{0}^{\otimes N}
$$

satisfy the asymptotics 3.2 for any $\mu \in \mathcal{M}_{1}(X)$ with rate functional $\tilde{I}(\mu)$ and $r_{N}=N$ iff the probability measures $\left(\delta_{N}\right)_{*} \mu_{\beta}^{(N)}$ on $\mathcal{M}_{1}(X)$ satisfy an LDP at speed $N$ with rate functional $I:=\tilde{I}-C_{\beta}$, where $C_{\beta}:=\inf _{\mu \in \mathcal{M}(X)} \tilde{I}(\mu)$.

Proof. Set $\tilde{\Gamma}_{N}:=\left(\delta_{N}\right)_{*} e^{-\beta H^{(N)}} \mu_{0}^{\otimes N}$ and $C_{N, \beta}:=-\frac{1}{N} \log Z_{N, \beta}$. By assumption $C_{N, \beta}$ is uniformly bounded and we denote by $C_{\beta}$ a given limit point of the sequence obtained by replacing $N$ with a subsequence $N_{j}$. Since $\frac{1}{N} \log \Gamma_{N}\left(B_{\epsilon}(v)\right)=\frac{1}{N} \log \tilde{\Gamma}_{N}\left(B_{\epsilon}(v)\right)+C_{N, \beta}$ we obtain that after replacing $N$ with the subsequence $N_{j}$ the probability measures $\Gamma_{N}$ satisfy (by Prop 3.3) an LDP with rate functional $\tilde{I}-C_{\beta}$. As a consequence $0=$ $\inf \left(\tilde{I}-C_{\beta}\right)$, showing that $C_{\beta}$ is independent of the subsequence. Hence, the whole sequence converges towards $C_{\beta}$, which proves one direction in the Lemma. The converse is proved in a similar way.

We will also use the following classical result of Sanov, which is the standard example of a LDP for point processes [30] (the result follow, for example, from the Gärtner-Ellis theorem; see Sect. 4.2).

Proposition 3.5. Let $X$ be a topological space and $\mu_{0}$ a finite measure on $X$. Then the law $\Gamma_{N}$ of the empirical measures of the corresponding Gibbs measure $\mu_{0}^{\otimes N}$ (i.e. $\left.H^{(N)}=0\right)$ satisfies an LDP with speed $N$ and rate functional the relative entropy $D_{\mu_{0}}$. 
We recall that the relative entropy $D_{\mu_{0}}$ (also called the Kullback-Leibler divergence or the information divergence in probability and information theory) is the functional on $\mathcal{M}_{1}(X)$ defined by

$$
D_{\mu_{0}}(\mu):=\int_{X} \log \frac{\mu}{\mu_{0}} \mu,
$$

when $\mu$ has a density $\frac{\mu}{\mu_{0}}$ with respect to $\mu_{0}$ and otherwise $D_{\mu_{0}}(\mu):=\infty$. When $\mu_{0}$ is a probability measure, $D_{\mu_{0}}(\mu) \geq 0$ and $D_{\mu_{0}}(\mu)=0$ iff $\mu=\mu_{0}$ (by Jensen's inequality).

3.2.1. Legendre-Fenchel transforms. Let $f$ be a function on a topological vector space $V$. Then its The Legendre-Fenchel transform is defined as following convex lower semicontinuous function $f^{*}$ on the topological dual $V^{*}$

$$
f^{*}(w):=\sup _{v \in V}\langle v, w\rangle-f(v)
$$

in terms of the canonical pairing between $V$ and $V^{*}$. In the present setting we will take $V=C^{0}(X)$ and $V^{*}=\mathcal{M}(X)$, the space of all signed Borel measures on a compact topological space $X$. We will use the following variant of the Brøndsted-Rockafellar property $A^{*}[25]$ :

Lemma 3.6. Let $f$ be function on $C^{0}(X)$ which is Gateaux differentiable. Then, for any $\mu \in \mathcal{M}(X)$ such that $f^{*}(\mu)<\infty$ there exists a sequence of $u_{j} \in C^{0}(X)$ such that

$$
\mu_{j}:=d f_{\mid u_{j}} \rightarrow \mu, \quad f^{*}\left(\mu_{j}\right) \rightarrow f^{*}(\mu)
$$

Proof. First recall that a convex function $g$ on a topological vector space $E$ is said to be subdifferentiable at $x \in E$ if $g(x)<\infty$ and $g$ admits a subgradient $x^{*}$ at $x$, i.e. an element $x^{*}$ in the topological dual $E^{*}$ such that for any $y \in E$

$$
g(y) \geq g(x)+\left\langle(y-x), x^{*}\right\rangle
$$

The set of all such subgradients is denoted by $(\partial g)(x)$. Now assume that $g=f^{*}$ for a convex function $f$ on a Banach space $V$. Then $g$ is a lower semi-continuous function convex function on the topological vector space $E:=V^{*}$ equipped with its weak topology. According to [25, Thm 2] any element $\mu \in V^{*}$ such that $f^{*}(\mu)<\infty$ has the property that there exists a sequence $\mu_{j} \rightarrow \mu$ in $V^{*}$ such that $f^{*}\left(\mu_{j}\right) \rightarrow f^{*}(\mu)$ and $f^{*}$ is subdifferentiable at $\mu_{j}$ with a subgradient in $V$. The latter property equivalently means that there exists $u_{j} \in V$ such that $\mu_{j} \in(\partial f)\left(u_{j}\right)$ (as follows from the definition of the Legendre-Fenchel transform). The proof is now concluded by setting $V:=C^{0}(X)$ and observing that if $f$ is Gateaux differentiable at $u \in V$, then $(\partial f)(u)=\left\{d f_{\mid u}\right\}$ (as is seen by restricting $f$ to any affine line).

Remark 3.7. By convexity, if $\mu=d f_{\mid u}$ for some $u \in V:=C^{0}(X)$, then $f^{*}(\mu)=$ $\left\langle u, d f_{\mid u}\right\rangle-f(u)$, which is essentially the classical definition of the Legendre transform of $f$ at $\mu$. Accordingly, the previous lemma may be reformulated as the statement that the Legendre-Fenchel transform is the greatest lower semi-continuous extension to all of $V^{*}$ of the Legendre transform, originally defined on $(d f)(V) \subset V^{*}$. 


\subsection{The proof of Theorem 5.7. We start with the following simple}

Lemma 3.8. Assume that $H^{(N)}$ satisfies the quasi-superharmonicity assumption in the second point of Theorem 1.1. Then, for any sequence of positive numbers $\beta_{N} \rightarrow \infty$

$$
-\mathcal{F}_{\beta_{N}}(u):=\frac{1}{N \beta_{N}} \log \int_{X^{N}} e^{-\beta_{N}\left(H^{(N)}+u\right)} d V^{\otimes N}=-\inf _{X^{N}} \frac{H^{(N)}+u}{N}+o(1)
$$

Proof. The inequality $\leq$ is trivial and to prove the reversed inequality we fix a sequence of $x^{(N)} \in X^{N}$ realizing the infimum appearing the right hand side above. Then replacing the integral of $X^{N}$ with an integral over the $L^{\infty}$-ball $B_{\epsilon}:=\left\{\left(x_{1}, \ldots, x_{N}\right): d_{g}\left(x, x_{i}^{(N)}\right) \leq\right.$ $\epsilon\}^{N}$, for a fixed number $\epsilon$ and a fixed metric $g$ with distance function $d_{g}$, and using the classical submean inequality in each variable with a fixed multiplicative constant $C$ gives

$$
\int_{X^{N}} e^{-\beta_{N}\left(H^{(N)}+u\right)} d V^{\otimes N} \geq C^{N} e^{-\beta_{N}\left(H^{(N)}+u\right)\left(x^{(N)}\right)} \int_{B_{\epsilon}} d V^{\otimes N} e^{-N \beta_{N} \delta_{\epsilon}}
$$

$\delta_{\epsilon}$ is the modulus of continuity of $u$, tending to 0 as $\epsilon \rightarrow 0$. Finally, since $\int_{B_{\epsilon}} d V^{\otimes N} \geq$ $\left(C^{\prime} \epsilon\right)^{N}$ letting $N \rightarrow \infty$ concludes the proof.

To handle the case when $\beta_{N}=\beta+o$ (1) for a finite $\beta$ we will need to use the subexponential dependence on the dimensions of the multiplicative constant appearing in Theorem 2.1. To this end we first recall that, since $X$ is assumed compact, the weak topology on $\mathcal{M}_{1}(X)$ is metrized by the Wasserstein 2-metric $d$ induced by a given Riemannian metric $g$ on $X$, where

$$
d(\mu, v)^{2}:=\inf _{\Gamma \in \Gamma(\mu, v)} \int d_{g}(x, y)^{2} d \Gamma,
$$

where $\Gamma(\mu, v)$ is the space of all couplings between $\mu$ and $\nu$, i.e. all probability measures $\Gamma$ on $X \times X$ such that the push forward of $\Gamma$ to the first and second factor is equal to $\mu$ and $\nu$, respectively.

Proposition 3.9. For any given $\epsilon>0$ there exists a positive constant $C>0$ such that the following submean inequality holds on $X^{N}$, for any $N$ :

$$
e^{\left.-\beta H^{(N)}\right)}\left(x^{(N)}\right) \leq C e^{C \epsilon N} \frac{\int_{B_{\epsilon}\left(x^{(N)}\right)} e^{-\beta H^{(N)}} d V^{\otimes N}}{\int_{B_{\epsilon^{2}}\left(x^{(N)}\right)} d V^{\otimes N}},
$$

where $B_{r}\left(x^{(N)}\right)$ denotes the inverse image in $X^{N}$, under the map $\delta_{N}$, of the Wasserstein ball of radius $r$ centered at $\delta_{N}\left(x^{(N)}\right)$

Proof. First observe that the pull-back of $d$ on $\mathcal{M}_{1}(X)$ to the quotient space $X^{(N)}:=$ $X^{N} / S_{N}$ under the map $\delta_{N}$ defined by the empirical measure (formula 3.1) coincides with $1 / N^{1 / 2}$ times the quotient distance function on $X^{(N)}$, induced by the product Riemannian metric on $X^{N}$ :

$$
\delta_{N}^{*} d=\frac{1}{N^{1 / 2}} d_{X^{(N)}}:=d^{(N)}
$$


Indeed, this is well-known and follows from the Birkhoff-Von Neumann theorem which gives that for any symmetric function $c(x, y)$ on $X \times X$ we have that if $\mu=\frac{1}{N} \sum_{i=1}^{N} \delta_{x_{i}}$ and $v=\frac{1}{N} \sum_{i=1}^{N} \delta_{y_{i}}$ for given $\left(x_{1}, \ldots, x_{N}\right),\left(y_{1}, \ldots, y_{N}\right) \in X^{N}$, then

$$
\inf _{\Gamma(\mu, \nu)} \int c(x, y) d \Gamma=\inf _{\Gamma_{N}(\mu, v)} \int c(x, y) d \Gamma
$$

where $\Gamma_{N}(\mu, v) \subset \Gamma(\mu, v)$ consists of couplings of the form $\Gamma_{\sigma}:=\frac{1}{N} \sum \delta_{x_{i}} \otimes \delta_{y_{\sigma(i)}}$, for $\sigma \in S_{N}$, where $S_{N}$ is the symmetric group on $N$ letters.

Now consider the metric space $\left(X^{(N)}, d^{(N)}\right)$ which is the quotient space defined with respect to the finite group $S_{N}$ acting isometrically on the Riemannian manifold $\left(X^{N}, g_{N}\right)$, where $g_{N}$ denotes $1 / N$ times the product Riemannian metric. By assumption $H^{(N)}$ is $S_{N}$-invariant and $\Delta_{g_{N}} H^{(N)} \leq C$ on $X^{N}$ (using the obvious scaling of the Laplacian). Moreover, since $X$ is compact there exists a non-negative number $k$ such that Ric $g \geq-k g$ on $X$ and hence rescaling gives Ric $g_{N} \geq-k N g_{N}$ on $\left(X^{N}, g_{N}\right)$. But the dimension of $X^{N}$ is equal to $n N$ and hence the assumptions in Theorem 2.1 are satisfied for $u:=e^{-\beta H^{(N)}}$ and $(X, g)$ replaced by $\left(X^{N}, g_{N}\right)$ with a constant $\kappa$ independent of $N$. Applying the latter theorem with $\delta=\epsilon$ and using the pull-back property in formula 3.7 then shows that the submean property 3.6 indeed holds.

We will also rely on the following simple but very useful lemma (which was used in the similar context of Fekete points in [13]).

Lemma 3.10. Fix $u_{*} \in C^{0}(X)$ and assume that $x_{*}^{(N)} \in X^{N}$ is a minimizer of the function $\left(H^{(N)}+u_{*}\right) / N$ on $X^{N}$. If the corresponding large $N$-limit $\mathcal{F}(u)$ exists for all $u \in C^{0}(X)$ and $\mathcal{F}$ is Gateaux differentiable at $u_{*}$, then $\delta_{N}\left(x_{*}^{(N)}\right)$ converges weakly towards $\mu_{*}:=d \mathcal{F}_{\mid u_{*}}$.

Proof. Fix $v \in C^{0}(X)$ and a real number $t$. Let $f_{N}(t):=\frac{1}{N}\left(H^{(N)}+u+t v\right)\left(x_{*}^{(N)}\right)$ and $f(t):=\mathcal{F}(u+t v)$. By assumption $\lim _{N \rightarrow \infty} f_{N}(0)=f(0)$ and $\liminf _{N \rightarrow} f_{N}(t) \geq$ $f(t)$. Note that $f$ is a concave function in $t$ (since it is defined as an inf of affine functions) and $f_{N}(t)$ is affine in $t$. But then it follows from the differentiability of $f$ at $t=0$ that $\lim _{N \rightarrow \infty} d f_{N}(t) / d t_{\mid t=0}=d f(t) / d t_{\mid t=0}$, i.e. that

$$
\lim _{N \rightarrow \infty}\left\langle\delta_{N}\left(x_{*}^{(N)}\right), v\right\rangle=\left\langle d \mathcal{F}_{\mid u}, v\right\rangle,
$$

which thus concludes the proof of the lemma (see [13, Lemma 3.1]).

The upper bound in the LDP. By Lemma 3.4 it will be enough to establish the LDP for the non-normalized measures $\Gamma_{N}$ in formula 3.3. To prove the upper bound of the integrals appearing in the equivalent formulation of the LDP in Prop 3.3 we fix a function $u \in C^{0}(X)$ and rewrite

$$
e^{-\beta H^{(N)}}=e^{-\beta\left(H^{(N)}+u\right)} e^{\beta u},
$$

Then, trivially, for any fixed $\epsilon>0$,

$$
\int_{B_{\epsilon}(\mu)} e^{-\beta H^{(N)}} d V^{\otimes N} \leq \sup _{B_{\epsilon}(\mu)}\left(e^{-\beta\left(H^{(N)}+u\right)}\right) \int_{B_{\epsilon}(\mu)} \mu_{u}^{\otimes N}, \mu_{u}:=e^{\beta u} d V
$$


Replacing the sup over $B_{\epsilon}(\mu)$ in the first factor above with the sup over all of $X^{N}$ and applying Sanov's theorem relative to the tilted volume form $\mu_{u}$ to the second factor gives

$$
\begin{aligned}
& \lim _{\epsilon \rightarrow 0} \limsup _{k \rightarrow \infty} \frac{1}{\beta N} \log \int_{B_{\epsilon}(\mu)} e^{-\beta H^{(N)}} d V^{\otimes N} \\
& \leq-\liminf _{N \rightarrow \infty} N^{-1} \inf _{X^{N}}\left(H^{(N)}+u\right)+\int u \mu-\frac{1}{\beta} D_{d V}(\mu),
\end{aligned}
$$

using that $D_{e^{\beta u} d V}(\mu)=-\beta \int u \mu+D_{d V}(\mu)$. According to Lemma 3.8 and the definition of the functional $\mathcal{F}(u)$ this means that

$$
\lim _{\epsilon \rightarrow 0} \limsup _{k \rightarrow \infty} \frac{1}{\beta N} \log \int_{B_{\epsilon}(\mu)} e^{-\beta H^{(N)}} d V^{\otimes N} \leq-\mathcal{F}(u)+\int u \mu-\frac{1}{\beta} D_{d V}(\mu),
$$

Finally, taking the infimum over all $u \in C^{0}(X)$ shows that the lim sup in the previous formula is bounded from above by $-F(\mu)$,

$$
F(\mu):=f^{*}(\mu)+\frac{1}{\beta} D_{d V}(\mu), \quad f(u):=-\mathcal{F}(-u)
$$

Remark 3.11. In the argument above $d V$ can be replaced by any finite measure $\mu_{0}$ on $X$.

The lower bound in the LDP. As usual, the proof of the lower bound in the LDP is the hardest. We first assume that

$$
\mu=d \mathcal{F}_{\mid u}
$$

for some $u \in C^{0}(X)$. Denote by $x^{(N)} \in X^{N}$ a sequence of minimizers of $H^{(N)}+u$. By Lemma 3.10 we have that

$$
\delta_{N}\left(x^{(N)}\right) \rightarrow \mu
$$

By the submean inequality 3.6

$$
\frac{1}{N} \log \Gamma_{N}\left(B_{2 \epsilon}(\mu)\right) \geq \frac{1}{N} \log \int_{B_{\epsilon^{2}}\left(x^{(N)}\right)} d V^{\otimes N}-\beta H^{(N)}\left(x^{(N)}\right) / N-\epsilon-\frac{C_{\epsilon}}{N}
$$

Since $\delta\left(x^{(N)}\right) \rightarrow \mu$ it follows that $\frac{1}{N} \log \Gamma_{N}\left(B_{2 \epsilon}(\mu)\right) \geq$

$$
\geq \frac{1}{N} \log \int_{B_{\epsilon^{2}\left(x^{(N)}\right)}} d V^{\otimes N}-\beta\left(H^{(N)}+u\right)\left(x^{(N)}\right) / N+\beta\langle u, \mu\rangle-\delta(\epsilon)-\epsilon-\frac{C_{\epsilon}}{N},
$$

where $\delta(\epsilon) \rightarrow 0$ as $\epsilon \rightarrow 0$. Moreover, fixing $\epsilon>0$ we may for $N$ sufficiently large assume that $B_{\epsilon^{2} / 2}(\mu) \subset B_{\epsilon^{2}}\left(\delta_{N}\left(x^{(N)}\right)\right)$ and hence letting $N \rightarrow \infty$ and using Sanov's theorem (i.e. Prop 3.5) for $\epsilon$ fixed and the convergence of $\left(H^{(N)}+u\right)\left(x^{(N)}\right) / N$ gives

$$
\liminf _{N \rightarrow \infty} \frac{1}{N} \log \Gamma_{N}\left(B_{2 \epsilon}(\mu)\right) \geq \beta\langle u, \mu\rangle-\delta(\epsilon)-\inf _{B_{\epsilon^{2} / 2}(\mu)} D_{d V}-\beta \mathcal{F}(u)-\epsilon
$$


(after perhaps replacing the original family $\delta(\epsilon)$ with a smaller one). Since $\mu$ is a candidate for the inf in the right hand side above the inf in question may be estimated from above by $D_{d V}(\mu)$ and hence letting $\epsilon \rightarrow 0$ concludes the proof under the assumption that $\mu:=d \mathcal{F}_{\mid u}$ for some $u \in C^{0}(X)$. To prove the general case we invoke Lemma 3.6 to write $\mu$ as a weak limit of $\mu_{j}:=d \mathcal{F}_{\mid u_{j}}$ for $u_{j} \in C^{0}(X)$. We may then replace $u$ in the previous argument with $u_{j}$ for a fixed $j$ and replace $\mu$ with $\mu_{j}$ in the previous argument to get, for $j \geq j_{\delta}, \liminf _{N \rightarrow \infty} \frac{1}{N} \log \Gamma_{N}\left(B_{3 \epsilon}(\mu)\right) \geq$

$$
\geq \liminf _{N \rightarrow \infty} \frac{1}{N} \log \Gamma_{N}\left(B_{2 \epsilon}\left(\mu_{j}\right)\right) \geq \beta\left\langle u_{j}, \mu_{j}\right\rangle-\delta_{j}(\epsilon)-\inf _{B_{\epsilon^{2} / 2}\left(\mu_{j}\right)} D_{d V}+\beta \mathcal{F}\left(u_{j}\right)-\epsilon
$$

But for $j$ sufficiently large $\mu_{j}$ is in the ball $B_{\epsilon^{2} / 2}(v)$ and hence the inf above is bounded from above by $D_{d V}(\mu)$ giving

$$
\liminf _{N \rightarrow \infty} \frac{1}{N} \log \Gamma_{N}\left(B_{3 \epsilon}(\mu)\right) \geq \beta\left\langle u_{j}, \mu_{j}\right\rangle-\delta_{j}(\epsilon)-D_{d V}(\mu)+\beta \mathcal{F}\left(u_{j}\right)-\epsilon
$$

Letting first $\epsilon \rightarrow 0$ and then $j \rightarrow \infty$ gives

$$
\liminf _{N \rightarrow \infty} \frac{1}{N} \log \Gamma_{N}\left(B_{3 \epsilon}(\mu)\right) \geq-\beta\left(\lim _{j \rightarrow \infty}\left(E\left(\mu_{j}\right)+\frac{1}{\beta} D_{d V}(\mu)\right)\right.
$$

Finally, by Lemma 3.6 we may assume that $E\left(\mu_{j}\right) \rightarrow E(\mu)$ and that concludes the proof.

The equation for the minimizer $\mu_{\beta}$. Finally, the Eq. 1.4 follows immediately from the following general convex analytical result:

Lemma 3.12. Let $X$ be a compact topological space and $f$ and $g$ be Gateaux differentiable convex functionals on $C^{0}(X)$ such that the differentials $d g$ and $d f$ takes values in $\mathcal{M}_{1}(X)$. Then

- The following identity holds:

$$
\inf _{\mathcal{M}_{1}(X)}\left(f^{*}+g^{*}\right)=\sup _{u \in C^{0}(X)}(-f(-u)-g(u))
$$

- if the sup in the right hand side above is attained at some $u_{0}$ in $C^{0}(X)$ (i.e. if $-f(-u)-$ $g(u)$ admits a critical point $\left.u_{0}\right)$, then, setting $\mathcal{F}(u):=-f(-u)$, the measure $\mu_{0}:=$ $d \mathcal{F}_{\mid u_{0}}$ minimizes the functional $f^{*}+g^{*}$ on $\mathcal{M}_{1}(X)$.

Proof. First observe that $f$ and $g$ are Lipschitz continuous on the Banach space $C^{0}(X)$. Indeed, setting $u_{t}:=u_{0}(1-t)+t u_{1}$, for $t \in[0,1]$, gives

$$
\left|f\left(u_{1}\right)-f\left(u_{0}\right)\right|=\left|\int_{0}^{1} d t \int_{X} d f_{u_{t}}\left(u_{1}-u_{0}\right)\right| \leq \sup _{X}\left|u_{1}-u_{0}\right|
$$

and similarly for $g$. The first point in the lemma is then obtained as a special case of the Fenchel-Rockafeller duality theorem which only requires that $f$ and $g$ be convex on a Banach space $V$ and that $f$ and $g$ be finite at some point $u$ where $f$ is moreover 
assumed continuous [24, Thm 1.12]. To prove the second point we let $u_{0}$ be a critical point of $\mathcal{F}(u)-g(u)$ on $C^{0}(X)$, i.e.

$$
d \mathcal{F}_{\mid u_{0}}=d g_{\mid u_{0}},
$$

which, by convexity, means that $u_{0}$ realizes the sup in the right hand side of formula 3.9. We rewrite,

$$
f^{*}(\mu):=\sup _{u \in C^{0}(X)}\langle u, \mu\rangle-f(u)=\sup _{u \in C^{0}(X)} \mathcal{F}(u)-\langle u, \mu\rangle
$$

(by replacing $u$ with $-u$ in the sup). Hence, if $\mu:=d \mathcal{F}_{\mid u}$ then, by concavity, $f^{*}(\mu):=$ $F(u)-\langle u, \mu\rangle$. Similarly, if $\mu=d g_{\mid v}$ then, by convexity, $g^{*}(\mu):=\langle u, \mu\rangle-g(u)$. All in all this means that if $u_{0}$ satisfies the critical point Eq. 3.10, then we can take $u=v=u_{0}$ to get

$$
f^{*}\left(\mu_{0}\right)+g^{*}\left(\mu_{0}\right)=\mathcal{F}\left(u_{0}\right)+0-g\left(u_{0}\right),
$$

which concludes the proof, using the first point.

\section{Relations to $\Gamma$-Convergence, the Gärtner-Ellis Theorem and Mean Energy}

Before turning to the applications of Theorem 1.1 in the complex geometric setting we explore some relations to previous results and methods in the literature.

4.1. Relations to Gamma-convergence. We recall that a sequence of functions $E_{N}$ on a topological space $\mathcal{P}$ is said to $\Gamma$-converge to a function $E$ on $\mathcal{P}$ if

$$
\begin{gathered}
\mu_{N} \rightarrow \mu \text { in } \mathcal{P} \\
\forall \mu
\end{gathered} \quad \exists \mu_{N} \rightarrow \mu \operatorname{in} \mathcal{P}: \quad \liminf _{N \rightarrow \infty} E_{N}\left(\mu_{N}\right) \geq E(\mu)
$$

(such a sequence $\mu_{N}$ is called a recovery sequence); see [23]. It then follows that $E$ is lower semi-continuous on $\mathcal{P}$. In the present setting we take, as before, $\mathcal{P}=\mathcal{M}(X)$ and define $E_{N}$ by setting $E_{N}=\infty$ on the complement of the image of the map $\delta_{N}$ and

$$
E_{N}\left(\delta_{N}\left(x_{1}, \ldots, x_{N}\right):=H^{(N)}\left(x_{1}, \ldots, x_{N}\right) / N\right.
$$

We can now formulate the following variant of Theorem 1.1:

Theorem 4.1. Let $H^{(N)}$ be a sequence of lower semi-continuous symmetric functions on $X^{N}$, where $X$ is a compact Riemannian manifold. Assume that

- The functions $E_{N}$ on $\mathcal{M}_{1}(X)$ determined by $H^{(N)}$ converge to a function $E$, in the sense of $\Gamma$-convergence on $\mathcal{M}_{1}(X)$.

- $H^{(N)}$ is uniformly quasi-superharmonic, i.e. $\Delta_{x_{1}} H^{(N)}\left(x_{1}, x_{2}, \ldots x_{N}\right) \leq C$ on $X^{N}$

Then, for any sequence of positive numbers $\left.\left.\beta_{N} \rightarrow \beta \in\right] 0, \infty\right]$ the measures $\Gamma_{N}:=$ $\left(\delta_{N}\right)_{*} e^{-\beta_{N} H^{(N)}}$ on $\mathcal{M}_{1}(X)$ satisfy, as $N \rightarrow \infty$, a LDP with speed $\beta_{N} N$ and good rate functional

$$
F_{\beta}(\mu)=E(\mu)+\frac{1}{\beta} D_{d V}(\mu)
$$


Proof. Using the characterization of a LDP in Proposition 3.3, the upper bound in the LDP follows almost immediately from the liminf property of the Gamma-convergence together with Sanov's theorem. To prove the lower bound fix $\mu \in \mathcal{M}_{1}(X)$ and take a recovery sequence $\mu_{N}$ corresponding to a sequence $x^{(N)} \in X^{N}$. Then, using the same notation for the balls as in the proof of Theorem 1.1 we have, for $\epsilon>0$ fixed and $N$ large,

$\int_{B_{2 \epsilon}(\mu)} e^{-\beta H^{(N)}} d V^{\otimes N} \geq \int_{B_{\epsilon}\left(x^{(N)}\right)} e^{-\beta H^{(N)}} d V^{\otimes N} \geq e^{N C \epsilon} e^{-N \beta E_{N}\left(\mu_{N}\right)} \int_{B_{\epsilon^{2}}\left(x^{(N)}\right)} d V^{\otimes N}$,

using the submean inequality in Theorem 2.1 in the last inequality. Letting first $N \rightarrow \infty$ and then $\epsilon \rightarrow 0$ then concludes the proof, using Sanov's theorem again.

It should be stressed that, in general, the functional $E(\mu)$ in the previous theorem will not be convex and hence the subset $\mathcal{C}_{\beta} \subset \mathcal{M}_{1}(X)$ consisting of the minima of $F_{\beta}$ will, in general, consist of more than one element. By general principles the LDP then implies that any limit point $\Gamma_{\infty} \in \mathcal{M}_{1}\left(\mathcal{M}_{1}(X)\right)$ of the laws $\Gamma_{N}$ is concentrated on $\mathcal{C}_{\beta}$ (in the terminology of statistical mechanics $\Gamma_{\infty}$ is thus a mixed state defined as a superposition of the pure states $\delta_{\mu}$ where $\mu \in \mathcal{C}_{\beta}$ ).

Remark 4.2. The proof of the previous theorem in the case $\beta=\infty$ is much simpler as it is does not require the sub-exponential dependence on the dimension in the submean inequality in Theorem 2.1. Indeed, the rough exponential bound used in the proof of Lemma 3.8 is enough. Moreover, all that is used in the proof for $\beta<\infty$ is that $\Delta_{x_{1}}\left(e^{-\beta_{N} H^{(N)}}\right) \geq-\lambda_{\beta} e^{-\beta_{N} H^{(N)}}$ for a constant $\lambda_{\beta}$ independent on $N$ (but the assumption that $\Delta_{x_{1}} H^{(N)} \leq C$ is a convenient way of ensuring that the previous inequality holds for any $\beta$ ).

Example 4.3. In the case when $X=\mathbb{R}^{n}$ equipped with the Euclidean distance it is known that the mean field Hamiltonian with pair interaction of the form $W(x, y)=w(|x-y|)$ (formula 1.14) $\Gamma$-convergences towards $E(\mu):=\int_{X^{2}} W \mu \otimes \mu$, if $w$ is lower semicontinuous and increasing close to 0 (see [48, Prop 2.8, Remark 2.19] and [15,17,28] for similar results). The proof exploits the explicit nature of $E(\mu)$ and a similar argument applies on a compact manifold when $W$ is continuous away from the diagonal with a singularity of the local form $w(|x-y|)$ close to the diagonal (compare [55,56]).

In contrast to the previous example, for the "determinantal" Hamiltonian 5.16 appearing in the complex geometric setting there is no explicit candidate for a limit $E(\mu)$. Instead the Gamma convergence is a consequence of the following dual criterion.

4.1.1. A criterion for Gamma convergence using duality. Next we separate out the convex analysis used in the proof of Theorem1.1 to get the following criterion for $\Gamma$ convergence:

Proposition 4.4. Let $E_{N}$ a sequence of functions on $\mathcal{M}_{1}(X)$ and assume that

$$
\lim _{N \rightarrow \infty} E_{N}^{*}(u)=f(u)
$$

where $f$ is a Gateaux differentiable convex function on $C^{0}(X)$. Then $E_{N}$ converges to $E:=f^{*}$ in the sense of $\Gamma$-convergence on the space $\mathcal{M}_{1}(X)$, equipped with the weak topology. 
Proof. First suppose that $\mu_{N} \rightarrow \mu$ weakly in $\mathcal{M}_{1}(X)$. Fix $u$ in $C^{0}(X)$. Then $-E_{N}\left(\mu_{N}\right)=$ $\left\langle u, \mu_{N}\right\rangle-E_{N}\left(\mu_{N}\right)-\langle u, \mu\rangle+o(1)$ and hence taking the sup over all $\mu \in \mathcal{M}_{1}(X)$ gives

$$
-E_{N}\left(\mu_{N}\right) \leq f_{N}(u)-\langle u, \mu\rangle+o(1)=f(u)-\langle u, \mu\rangle+o(1) .
$$

Finally, letting first $N \rightarrow \infty$ and then taking the sup over all $u \in C^{0}(X)$ concludes the proof of the lower bound for $E_{N}\left(\mu_{N}\right)$.

To prove the existence of a recovery sequence we first assume that $\mu=d f_{\mid u_{\mu}}$ for some $u_{\mu} \in C^{0}(X)$. Then,

$$
f^{*}(\mu)=\left\langle u_{\mu}, \mu\right\rangle-f\left(u_{\mu}\right)=\left\langle u_{\mu}, \mu\right\rangle-f_{N}\left(u_{\mu}\right)+o(1),
$$

Now, by the weak compactness of $\mathcal{M}_{1}(X)$ the sup defining $f_{N}$ is attained at some $\mu_{N} \in \mathcal{M}_{1}(X)$ and hence

$$
f^{*}(\mu)+o(1)=\left\langle u_{\mu}, \mu\right\rangle-\left(\left\langle u_{\mu}, \mu_{N}\right\rangle-E_{N}\left(\mu_{N}\right)\right)
$$

Next, by a minor generalization of Lemma $3.10 \mu_{N} \rightarrow \mu\left(:=d f_{\mid u_{\mu}}\right)$ and hence $f^{*}(\mu)=$ $0+E_{N}\left(\mu_{N}\right)+o(1)$, as desired. Finally, the proof of the existence of recovery sequence for any $\mu$ such that $E(\mu)<\infty$ is concluded by a simple diagonal argument based on Lemma 3.6 applied to $E:=f^{*}$.

Now, if $E_{N}$ is of the form 4.2, then

$$
f_{N}(u):=\sup _{X^{N}} \frac{1}{N} u\left(x_{1}\right)+\ldots+\frac{1}{N} u\left(x_{N}\right)-\frac{1}{N} H^{(N)}\left(x_{1}, \ldots, x_{N}\right)
$$

Thanks to the previous proposition the first assumption in Theorem 1.1 thus implies (also using Lemma 3.8) that $E_{N} \rightarrow E$ in the sense of $\Gamma$-convergence on $\mathcal{M}(X)$. Accordingly we recover Theorem 1.1 from Theorem 4.1.

Remark 4.5. In general, if $E_{N}$ gamma converges to a function $E$ on $\mathcal{M}_{1}(X)$, then it follows (almost directly) that $E_{N}^{*} \rightarrow E^{*}$ point-wise on $C^{0}(X)$. Hence, the point of the previous proposition is that it gives a converse statement under the assumption that $E^{*}$ is Gateaux differentiable. By basic convex duality it thus follows from the previous proposition that $E_{N}$ converges to a strictly convex functional $E$ on $\mathcal{M}_{1}(X)$ iff $E_{N}^{*} \rightarrow E^{*}$ point-wise on $C^{0}(X)$, with $E^{*}$ Gateaux differentiable.

4.2. Relations to the Gärtner-Ellis theorem. First observe that

$$
\int_{X^{N}} e^{-\beta_{N}\left(H^{(N)}+u\right)} d V^{\otimes N}=\widehat{\Gamma_{N}}\left(-r_{N} u\right),
$$

where $\Gamma_{N}$ is the measure

$$
\Gamma_{N}:=\left(\delta_{N}\right)_{*}\left(e^{-\beta H^{(N)}} d V^{\otimes N}\right)
$$

on $\mathcal{M}_{1}(X)$ and $\widehat{\Gamma_{N}}$ denotes its Laplace transform on $C^{0}(X)$. In this context the GärtnerEllis theorem may be formulated as follows (see [30, Cor 4.6.14, p. 148] and references therein): 
Theorem 4.6 (Gärtner-Ellis). Let $H^{(N)}$ be a sequence of Hamiltonians on $X^{N}$ and $\beta_{N}$ a sequence of positive numbers such that $\left.\left.\beta_{N} \rightarrow \beta \in\right] 0, \infty\right]$. Assume that, for any $u \in C^{0}(X)$, as $N \rightarrow \infty$,

$$
\mathcal{F}_{\beta_{N}}(u):=-\frac{1}{N \beta_{N}} \log \int_{X^{N}} e^{-\beta_{N}\left(H^{(N)}+u\right)} d V^{\otimes N} \rightarrow \mathcal{F}_{\beta}(u),
$$

where $\mathcal{F}$ is a Gateaux differentiable function. Then the measures $\Gamma_{N}$ on $\mathcal{M}_{1}(X)$ satisfy, as $N \rightarrow \infty$, a LDP with speed $\beta_{N} N$ and good rate functional $f^{*}(\mu)$, where $f(u):=$ $-\mathcal{F}(-u)$.

Compared with the Gärtner-Ellis theorem the main point of Theorem 1.1 is thus that the assumption that the convergence of the partition functions in formula 4.5 holds for $\beta=\infty$ is enough to ensure that one gets an LDP for any $\beta \in] 0, \infty$ [ (under the quasi-subharmonicity assumption). As a consequence, the convergence of the partition functions then also hold for any $\beta \in] 0, \infty$ [ with the limiting functional $-\mathcal{F}_{\beta}(\cdot)$ defined as the Legendre-Fenchel transform of the rate functional $F_{\beta}$ appearing in Theorem 1.1. In fact, the latter convergence is equivalent to the LDP in question, as made precise by the following

Lemma 4.7. Let $H^{(N)}$ be a sequence of Hamiltonians on $X^{N}$ and $\beta_{N}$ a sequence of positive numbers such that $\left.\beta_{N} \rightarrow \beta \in\right] 0, \infty[$. Assume that, for any given volume form $d V$, the corresponding partition functions $Z_{N . \beta_{N}}$ satisfy

$$
\lim _{N \rightarrow \infty}-\frac{1}{N \beta_{N}} \log Z_{N . \beta_{N}}:=\inf _{\mu} F_{\beta}, \quad F_{\beta}:=E+D_{d V} / \beta,
$$

with $E(\mu)$ convex. Then the measures $\left(\delta_{N}\right)_{*}\left(e^{-\beta H^{(N)}} d V^{\otimes N}\right)$ on $\mathcal{M}_{1}(X)$ satisfy, as $N \rightarrow \infty$, an LDP with speed $\beta_{N} N$ and good rate functional $F_{\beta}$. Moreover, if the asymptotics above also holds for $\beta=\infty$ with $E(\mu)$ strictly convex, then the LDP holds for $\beta=\infty$, as well.

Proof. Fixing a volume form $d V$ and applying the asymptotics in the lemma to the volume forms $e^{-\beta u} d V$ for any $u \in C^{0}(X)$ reveals that the asymptotics 4.5 hold with $f_{\beta}$ given by the Legendre-Fenchel transform of $E+D_{d V} / \beta$. Now, if $E$ is convex, then $E+D_{d V} / \beta$ is strictly convex (since $D_{d V}$ is) and hence it follows from basic convex duality that $f_{\beta}$ is Gateaux differentiable. In fact, the differential $\mu_{u}:=d f_{\beta \mid u}$ is the unique minimizer attaining the sup defining $f_{\beta}(u)$, viewed as the Legendre-Fenchel transform of $E+D_{d V} / \beta$. Equivalently, $\mu_{u}$ is the unique minimizer of the strictly convex functional $\mu \mapsto E(\mu)+\langle u, \mu\rangle+D_{d V}(\mu) / \beta$.

Remark 4.8. Let $\beta_{N}$ be sequence tending to $\infty$. By convex duality the Gärtner-Ellis theorem may in the present setting, be formulated as follows (also using Varadhan's lemma [30] in the converse): let $E_{N}$ be a sequence of functions on $\mathcal{M}_{1}(X)$. Then $e^{-\beta_{N} N E_{N}}\left(\delta_{N}\right)_{*}\left(d V^{\otimes N}\right) \sim e^{-\beta_{N} N E(\mu)}$ in the sense of a LDP, with $E(\mu)$ strictly convex iff $\beta_{N} N$ times the log of the Laplace transform of $e^{-\beta_{N} N E_{N}}\left(\delta_{N}\right)_{*}\left(d V^{\otimes N}\right)$ converges to the Gateaux differentiable function $E^{*}$ on $C^{0}(X)$. 
4.3. Relations to the existence of the mean energy. Given a sequence of Hamiltonians $H^{(N)}$ on $X^{N}$ we set

$$
\bar{E}_{N}(\mu):=\frac{1}{N} \int_{X^{N}} H^{(N)} \mu^{\otimes N}
$$
$\bar{E}(\mu)$.

If the limit as $N \rightarrow \infty$ exists then we will call it the mean energy of $\mu$, denoted by

Example 4.9. If $H^{(N)}$ is the mean field Hamiltonian associated to the pair interaction potential $W$ (formula emph1.14) then, trivially, $E(\mu)=\bar{E}_{N}(\mu)$ for any $\mu$ such that $W \in L^{1}(\mu)$.

It follows immediately from the definition that if the limit of $E_{N}^{*}\left(:=f_{N}\right)$ appearing in formula 4.4 exists then

$$
\bar{E}(\mu) \geq f^{*}(\mu)
$$

(but, in general this is a strict inequality, for example if $\bar{E}(\mu)$ is not convex). In particular, under the assumptions in Theorem 1.1 we have $\bar{E}(\mu) \geq E(\mu)$, where $E(\mu)$ appears as the rate functional in Theorem 1.1 for $\beta=\infty$ (using Lemma 3.8). Motivated by the complex geometric applications discussed in Sect. 6 this leads one to consider the following

Problem 4.10. Show that the assumptions on $H^{(N)}$ in Theorem 1.1 imply that the corresponding mean energy $\bar{E}(\mu)$ exists when $\mu$ is a volume for (perhaps under additional appropriate assumptions on $\left.H^{(N)}\right)$.

As illustrated by the following lemma this problem turns out to be related to the asymptotics of the Gibbs measures with $\beta$ negative:

Lemma 4.11. Assume that there exists some negative $\beta_{0}$ such that for any $\beta \geq \beta_{0}$ the corresponding Gibbs measures are well-defined, i.e. $Z_{N . \beta_{N}}<\infty$ for $N$ sufficiently large. Moreover, assume that there exists a functional $E(\mu)$ such that

$$
-\lim _{N \rightarrow \infty} \frac{1}{N} \log Z_{N . \beta_{N}}=\inf _{\mu \in \mathcal{M}_{1}(X)} \beta E(\mu)+D_{d V}(\mu)>-\infty,
$$

for any volume form $d V$. Then the mean energy $\bar{E}(\mu)$ exists for any volume form $\mu$ and $\bar{E}(\mu)=E(\mu)$.

Proof. First observe that, by Jensen's inequality, the number $f_{N}(\beta):=-\frac{1}{N} \log Z_{N . \beta}$ appearing in the right hand side above for $N$ is concave in $\beta$ (and, by assumption, finite). Moreover, $\partial f_{N}(\beta) / \partial \beta=\bar{E}_{N}(d V)$ at $\beta=0$. Further more, the finite function $f(\beta)$ defined by the right hand side in formula 4.6 is also concave, as it is an infimum of a family of linear functions and for $\beta=0$ the infimum is attained precisely at $\mu=d V$. Hence, by basic convex analysis, the derivative of $f$ at $\beta=0$ exists and is given by $E(d V)$. Finally, the proof is concluded by using that if $f_{N}$ is a sequence of convex functions converging point-wise to convex function $f$ such that $f_{N}$ and $f$ are differentiable at 0 then the corresponding derivatives at 0 also converge. 
It should, however, be stressed that, if $H^{(N)}$ is too singular then the partition function $Z_{N . \beta}$ is equal to $\infty$, for any $\beta<0$ (even if $H^{(N)}$ is quasi-superharmonic as in the assumptions of Theorem 1.1). Indeed, for the mean field Hamiltonian corresponding to a pair interaction $W$ this happens as soon as $W$ has a repulsive power-law singularity, i.e. $W(x, y) \sim|x-y|^{\alpha}$ with $\alpha<0$ close to the diagonal. On the other hand, in the case of a logarithmic singularity $Z_{N . \beta_{N}}$ is indeed finite for $\beta_{0}<0$ and sufficiently close to 0 (see [22] for the corresponding LDP in the setting of the $2 \mathrm{D}$ vortex model).

Using the Gibbs variational principle some converses to Lemma 4.11 can be established [11], where the existence of the mean energy is assumed (and some additional assumptions), by extending the approach of Messer-Spohn [45]. However is should be stressed that the main point of our proof of Theorem 1.1 is that it does note rely on the existence of the mean energy $\bar{E}(\mu)$, which, as pointed above, is an open problem in the present setting.

\section{Applications to Kähler-Einstein Geometry}

In this section we will apply Theorem 1.1 to complex manifolds $X$ equipped with a line bundle $L$, assuming that $L$ is positive. The extension to big line bundles (and varieties of positive dimension) is given in the companion paper [9], using the full power of the pluripotential theory developed in $[7,14,20]$ (see the discussion in Sect. 5.4).

5.1. Kähler geometry setup. Let $X$ be an $n$-dimensional compact complex manifold and denote by $J$ the corresponding complex structure viewed as an endomorphism of the real tangent bundle satisfying $J^{2}=-I$.

5.1.1. Kähler forms/metrics. On a complex manifold $(X, J)$ anti-symmetric two forms $\omega$ and symmetric two tensors $g$ on $T X \otimes T X$, which are $J$-invariant, may be identified by setting

$$
g:=\omega(\cdot, J \cdot)
$$

Such a real two form $\omega$ is said to be Kähler if $d \omega=0$ and the corresponding symmetric tensor $g$ is positive definite (i.e. defines a Riemannian metric) ${ }^{2}$ Conversely, a Riemannian metric $g$ is said to be Kähler if it arises in this way (in Riemannian terms this means that parallel transport with respect to $g$ preserves $J$ ). By the local $\partial \bar{\partial}$-lemma a $J$-invariant two form $\omega$ is closed, i.e. $d \omega=0$ if and only if $\omega$ may be locally expressed as $\omega=\frac{i}{2 \pi} \partial \bar{\partial} \phi$, in terms of a local smooth function $\phi$ (called a local potential for $\omega$ ). In real notation this means that

$$
\omega=d d^{c} \phi, d^{c}:=-\frac{1}{4 \pi} J^{*} d
$$

(and hence $\omega$ is Kähler iff $\phi$ is strictly plurisubharmonic). The normalization above ensures that $d d^{c} \log |z|^{2}$ is a probability measure on $\mathbb{C}$. We will denote by $[\omega] \in H^{2}(X, \mathbb{R})$ the de Rham cohomology represented by $\omega$. If $\omega_{0}$ is a fixed Kähler form then, according to the global $\partial \bar{\partial}$-lemma, any other Kähler metric in $\left[\omega_{0}\right]$ may be globally expressed as

$$
\omega_{\varphi}:=\omega_{0}+d d^{c} \varphi, \quad \varphi \in C^{\infty}(X),
$$

\footnotetext{
2 In the complex analysis literature a $J$-invariant two form $\omega$ is usually said to be of type $(1,1)$ since $\omega=\sum_{i, j} \omega_{i j} d z_{i} \wedge d \bar{z}_{j}$ in local holomorphic coordinates.
} 
where $\varphi$ is determined by $\omega_{0}$ up to an additive constant. We set

$$
\mathcal{H}(X, \omega):=\left\{\varphi \in C^{\infty}(X): \omega_{\varphi}>0\right\}
$$

The association $\varphi \mapsto \omega_{\varphi}$ thus allows one to identify $\mathcal{H}(X, \omega) / \mathbb{R}$ with the space of all Kähler forms in $\left[\omega_{0}\right]$.

5.1.2. Metrics on line bundles and curvature. Let $L$ be a holomorphic line bundle on $X$ and $\|\cdot\|$ a Hermitian metric on $L$. The normalized curvature two form of $\|\cdot\|$ may be (locally) written as

$$
\omega:=-d d^{c} \log \|s\|^{2},
$$

in terms of a given local trivialization holomorphic section $s$ of $L$. The corresponding cohomology class $[\omega]$ is independent of the metric $\|\cdot\|$ on $L$ and coincides with the first Chern class $c_{1}(L)$ in $H^{2}(X, \mathbb{R}) \cap H^{2}(X, \mathbb{Z})$ (conversely, any such cohomology class is the first Chern class of line bundle $L$ ). A line bundle $L$ is said to be positive if it admits a metric with positive curvature, i.e. such that the curvature form $\omega$ is Kähler. Fixing a reference metric $\|\cdot\|$ on $L$ with curvature form $\omega_{0}$ any other metric on $L$ may be expressed as $\|\cdot\| e^{-u / 2}$, for $u \in C^{\infty}(X)$ and its curvature is positive iff $u \in \mathcal{H}(X, \omega)$. When $L$ is the canonical line bundle $K_{X}$, i.e. the top exterior power of the holomorphic cotangent bundle of $X$ :

$$
K_{X}:=\operatorname{det}\left(T^{*} X\right)
$$

any volume form $d V$ on $X$ induces a smooth metric $\|\cdot\|_{d V}$ on $K_{X}$, by locally setting $\|d z\|_{d V}^{2}:=c_{n} d z \wedge d \bar{z} / d V$, where $d z:=d z_{1} \wedge \cdots \wedge d z_{n}$ in terms of local holomorphic coordinates and $c_{n} d z \wedge d \bar{z}$ is a short hand for the local Euclidean volume form $\frac{i}{2} d z_{1} \wedge$ $d \bar{z}_{1} \wedge \cdots \wedge \frac{i}{2} d z_{n} \wedge d \bar{z}_{n}$. When $d V$ is the volume form of a given Kähler metric $\omega$ on $X$, i.e. $d V=\omega^{n} / n$ !, then its curvature form may be identified with minus the Ricci curvature of $\omega$, i.e.

$$
\operatorname{Ric} \omega=-d d^{c} \log \frac{d V}{c_{n} d z \wedge d \bar{z}} .
$$

By a slight abuse of notation we will also write Ric $(d V)$ for the right hand side in formula 5.1 .

5.1.3. Twisted Kähler-Einstein metrics. A Kähler metric $\omega_{\beta}$ is said to be a twisted Kähler-Einstein metric if it satisfies the twisted Kähler-Einstein equation

$$
\operatorname{Ric} \omega=-\beta \omega+\eta,
$$

where the form $\eta$ is called the twisting form. Since $\omega_{\beta}$ is Kähler the form $\eta$ is necessarily closed and $J$-invariant. The corresponding equation at the level of cohomology classes is

$$
[\eta]=-c_{1}\left(K_{X}\right)+\beta[\omega]
$$

Fixing, once and for all, a volume form $d V$ on $X$ gives the following one-to-one corresponds between twisting forms $\eta$ and Kähler forms $\omega_{0}$ solving the cohomological equation above:

$$
\eta:=\beta \omega_{0}+\operatorname{Ric} d V .
$$

The following lemma then follows directly from the expression 5.1 for the Ricci curvature of a Kähler metric: 
Lemma 5.1. Let $X$ be a compact complex manifold endowed with a J-invariant and closed form $\eta$. Then a Kähler form $\omega_{\beta}$ solves the corresponding twisted Kähler-Einstein equation 5.2 iff $\omega_{\beta}:=\omega_{0}+d d^{c} \varphi_{\beta}$ for a unique $\varphi_{\beta} \in \mathcal{H}(X, \omega)$ solving the PDE

$$
\omega_{\varphi}^{n}=e^{\beta \varphi} d V
$$

The celebrated Aubin-Yau theorem may now be formulated as follows:

Theorem 5.2 (Aubin-Yau). [3,53] Given a compact complex manifold X, endowed with a Kähler form $\omega_{0}$ and a volume form $d V$, the PDE 5.4 admits, for any positive number $\beta$, a unique solution $\varphi_{\beta} \in \mathcal{H}(X, \omega)$. Equivalently, given a $J$-invariant and closed form $\eta$ such that the class $[\eta]+c_{1}\left(K_{X}\right)$ is positive (i.e. contains a Kähler form) there exists a unique Kähler metric $\omega_{\beta}$ solving the twisted Kähler-Einstein equation 5.2.

Example 5.3. A complex manifold $X$ admits a Kähler-Einstein metric with negative Ricci curvature iff $K_{X}$ is positive (and the metric is unique). Indeed, if $K_{X}$ is positive then, by the very definition of positivity, we can take $\omega_{0}:=-\operatorname{Ric} d V$ for some volume form on $X$, ensuring that $\eta=0$ above, with $\beta=1$ (and the converse is trivial).

Remark 5.4. When $n \geq 2$ the equation is 5.2 precisely the trace-reversed formulation of Einstein's equations on $X$ (with Euclidean signature): $-\beta$ is the cosmological constant and $\eta$ is the trace-reversed stress-energy tensor. Here we are only concerned with the solutions which are Kähler metrics.

5.1.4. The projection operator $P_{\omega_{0}}$ to the space $P S H\left(X, \omega_{0}\right)$. Next, we recall the definition of the operator $P$ introduced in [12] (which turns out to be related to the limit as $\beta \rightarrow \infty$ of the equations 5.4). Given $u \in C^{0}(X)$ we set

$$
(P u)(x):=\sup _{\varphi \in \mathcal{H}\left(X, \omega_{0}\right)}\{\varphi(x): \varphi \leq u\}
$$

which defines an operator

$$
P: C^{0}(X) \rightarrow P S H\left(X, \omega_{0}\right)
$$

from $C^{0}(X)$ to the space $P S H\left(X, \omega_{0}\right)$ of all $\omega_{0}-$ psh functions on $X$, i.e. all upper semi-continuous functions $\varphi$ in $L^{1}(X)$ such that $\omega_{\varphi} \geq 0$ in the sense of currents. In fact, the operator $P$ defines a projection operator from $C^{0}(X)$ onto $P S H\left(X, \omega_{0}\right) \cap C^{0}(X)$. More generally, if $u \in C^{\infty}(X)$, the current $d d^{c}(P u)$ has coefficients in $L_{\text {loc }}^{\infty}$, i.e.

$$
\omega_{P u} \in L_{\mathrm{loc}}^{\infty}
$$

In fact, as shown in [10], taking $d V$ in Eq. 5.4 to be of the form $d V=e^{-\beta u} d V$ one has

$$
\lim _{\beta \rightarrow \infty} \varphi_{\beta}=P u
$$

uniformly on $X$ and with a uniform upper bound on the corresponding Kähler forms $\omega_{\varphi_{\beta}}$. 
5.1.5. The Monge-Ampère operator and the functionals $\mathcal{E}$ and $\mathcal{F}$. The second order operator

$$
\varphi \mapsto M A(\varphi):=\omega_{\varphi}^{n}
$$

appearing in the Eq. 5.4, is the complex Monge-Ampère operator (with respect to the reference form $\left.\omega_{0}\right){ }^{3}$ By Stokes theorem

$$
\int_{X} \omega_{\varphi}^{n}=\int_{X} \omega_{0}^{n}:=V
$$

which is hence a positive number independent of $\varphi \in C^{\infty}(X)$. Up to a trivial scaling we may and will assume that $V=1$. When $n=1$ the operator $M A$ may be identified with the Laplacian, but when $n \geq 2$ it is fully non-linear. The one-form on $C^{\infty}(X)$ defined by $M A$ is closed and hence admits a primitive $\mathcal{E}$, i.e. a functional on $C^{\infty}(X)$ whose differential is given by

$$
d \mathcal{E}_{\mid \varphi}=M A(\varphi)
$$

The functional $\mathcal{E}$ is only determined up to an additive constant which may be fixed by the normalization condition $\mathcal{E}(0)=0$.

Using pluripotential theory $[14,20]$ the operator $M A$ can be extended from $\mathcal{H}_{\omega_{0}}$ to all of $P S H\left(X, \omega_{0}\right)$ giving a positive measures satisfying

$$
\int_{X} M A(\varphi) \leq V(:=1)
$$

Similarly the functional $\mathcal{E}$ also extends from $\mathcal{H}\left(X, \omega_{0}\right)$ to an increasing lower-semi continuous functional on $\operatorname{PSH}\left(X, \omega_{0}\right)$. We then set

$$
\mathcal{F}(u):=(\mathcal{E} \circ P)(u)
$$

which by [12] defines a Gateaux differentiable functional on $C^{0}(X)$. More precisely,

$$
(d \mathcal{F})_{\mid u}=M A(P u)
$$

This setup leads to a direct variational approach for solving complex Monge-Ampère equations, including the Aubin-Yau equation 5.4, in the more general setting of big cohomology classes and singular volume forms $d V$ [14] (compare Sect. 5.4). However, in the present setting where $L$ is positive the pluripotential theory can be dispensed with by observing that $M A(\varphi)$ is a well-defined probability measure as long as $\omega_{\varphi}$ is in $L_{\mathrm{loc}}^{\infty}$ (using that $M A(\varphi)$ is point-wise defined almost everywhere on $X$ ). Then $\mathcal{F}(u)$ may be defined by first taking $u$ to be in $C^{\infty}$ and using the regularity result 5.6 for $P u$. One then defines $\mathcal{F}$ on $C^{0}(X)$ as the unique continuous extension of $\mathcal{F}$ from $C^{\infty}(X)$, using that $\mathcal{F}(u)$ is Lipschitz continuous on $C^{\infty}(X)$ with respect to the $C^{0}$-norm (as follows form general principles; see the beginning of the proof of Lemma 3.12).

\footnotetext{
3 The terminology stems from the fact that when $\omega_{0}=0$ (which can always be locally arranged by shifting $\varphi)$ the density of $M A(\varphi)$ is proportional to the determinant of the complex Hessian $\partial \bar{\partial} \varphi$.
} 
5.2. The "temperature deformed" determinantal point processes on $X$. Let $(X, L)$ be a polarized manifold, i.e. an $n$-dimensional complex compact manifold $X$ endowed with a positive holomorphic line bundle $L$. We will denote by $H^{0}(X, k L)$ the space of all global holomorphic sections with values in the $k$ th tensor power of $L$ (using additive notation for tensor powers). By the Hilbert-Samuel theorem

$$
N_{k}:=\operatorname{dim} H^{0}(X, k L)=V k^{n}+o\left(k^{n}\right),
$$

where $V=\int_{X} c_{1}(L)^{n}>0$.

To the data $\left(\|\cdot\|, d V, \beta_{k}\right)$ consisting of a Hermitian metric $\|\cdot\|$ on $L$, a volume form $d V$ on $X$ and a sequence of positive number $\beta_{k}$ we can associate the following sequence of symmetric probability measures on $X^{N_{k}}$ :

$$
\mu^{\left(N_{k}, \beta\right)}:=\frac{\left\|\left(\operatorname{det} S^{(k)}\right)\left(x_{1}, x_{2}, \ldots x_{N_{k}}\right)\right\|^{2 \beta_{k} / k} d V^{\otimes N_{k}}}{Z_{N_{k}, \beta}}
$$

where det $S^{(k)}$ is a generator of the top exterior power $\Lambda^{N_{k}} H^{0}(X, k L)$, viewed as a one-dimensional subspace of $H^{0}\left(X^{N_{k}},(k L)^{\bigotimes N_{k}}\right)$ under the usual isomorphism between $H^{0}\left(X^{N_{k}},(k L)^{\bigotimes N_{k}}\right)$ and the $N_{k}$-fold tensor product of $H^{0}(X, k L)$. The number $Z_{N_{k}, \beta}$ is the normalizing constant

$$
Z_{N_{k}, \beta}:=\int_{X^{N_{k}}}\left\|\operatorname{det} S^{(k)}\right\|^{2 \beta / k} d V^{\otimes N_{k}}
$$

By homogeneity the probability measure $\mu^{\left(N_{k}, \beta\right)}$ is independent of the choice of generator det $S^{(k)}$ and thus only depends on the data $\left(\|\cdot\|, d V, \beta_{k}\right)$. We will refer to to the corresponding random point processes on $X$, as the temperature deformed determinantal point processes on $X$ attached to $\left(\|\cdot\|, d V, \beta_{k}\right.$ ) (the special case $\beta_{k}=k$ defines a bona fide determinantal point process, as recalled below).

Remark 5.5. Since the transformation $\left(\|\cdot\|, d V, \beta_{k}\right) \mapsto\left(\|\cdot\| e^{-u / 2}, e^{u \beta_{k}} d V, \beta_{k}\right)$, for $u \in C^{0}(X)$, leaves the probability measure 5.11 invariant, the processes above only depend on the data $\left(\|\cdot\|, d V, \beta_{k}\right)$ through the corresponding two form $\eta$, defined by formula 5.3. Moreover, any twisting form $\eta$ such that the cohomology class $([\eta]+$ $\left.c_{1}\left(K_{X}\right)\right) / \beta_{k}$ defines a positive class in $H^{2}(X, \mathbb{R}) \cap H^{2}(X, \mathbb{Z})$ arises from a suitable choice of data $\left(\|\cdot\|, d V, \beta_{k}\right)$ (compare Sect. 5.1.3).

It will be convenient to take det $S^{(k)}$ to be the generator determined by a basis $s_{1}, \ldots, s_{N_{k}}$ in $H^{0}(X, k L)$ which is orthonormal with respect to the $L^{2}$-product determined by $(\|\cdot\|, d V)$ for any fixed volume form $d V$ on $X$ :

$$
\langle s, s\rangle_{L^{2}}:=\int_{X}\|s\|^{2} d V
$$

We then take $\left(\operatorname{det} S^{(k)}\right)\left(x_{1}, x_{2}, \ldots, x_{N_{k}}\right):=$

$$
=\operatorname{det}\left(s_{i}\left(x_{j}\right)\right):=\sum_{\sigma \in S_{N_{k}}}(-1)^{\operatorname{sign}(\sigma)} s_{1}\left(x_{\sigma(1)}\right) \cdots s_{N_{k}}\left(x_{\sigma\left(N_{k}\right)}\right)
$$


Example 5.6. The model case of a polarized manifold is $(X, L)=\left(\mathbb{P}^{m}, \mathcal{O}(1)\right)$, where $\mathbb{P}^{m}\left(:=\mathbb{C}^{m+1}-\{0\}\right) / \mathbb{C}^{*}$ is $m$-dimensional complex projective space and $\mathcal{O}(1)$ is the hyperplane line bundle over $\mathbb{P}^{m}$ (the model positively curved metric on $\mathcal{O}(1)$ is the Fubini-Study metric induced from the Euclidean metric on $\mathbb{C}^{m+1}$ ). More generally, taking $X$ to be a non-singular algebraic variety of $\mathbb{P}^{m}$ and $L$ as the restriction to $X$ of $\mathcal{O}(1)$ gives a polarized where the elements in $H^{0}(X, k L)$ are, for $k$ sufficiently large, the restrictions to $X$ of homogeneous polynomials of degree $k$ on $\mathbb{P}^{m}$ (in fact, by the Kodaira embedding theorem any polarized manifold $(X, L)$ may, after replacing $L$ with a sufficiently high tensor power, be concretely realized as $\left(X, \mathcal{O}(1)_{\mid X}\right)$ ). In the case of $X=\mathbb{P}^{1}$ (=the Riemann sphere) with $\|\cdot\|$ denoting the Fubini-Study metric on $\mathcal{O}(1)$ whose curvature form $\omega_{0}$ is the invariant measure on $\mathbb{P}^{1}$ one can take the base $\left\{s_{i}\right\}$ to consist of monomials and factorize

$$
\left\|\operatorname{det} S^{(k)}\right\|\left(x_{1}, x_{2}, \ldots, x_{N}\right)=Z_{N} \prod_{1 \leq i<j \leq N}\left|x_{i}-x_{j}\right|,
$$

where $N=k+1$ and $X$ has been identified with the unit-sphere in Euclidean $\mathbb{R}^{3}$ and where $Z_{N}=N^{N}\left(\begin{array}{c}N-1 \\ 0\end{array}\right) \ldots\left(\begin{array}{c}N-1 \\ N-1\end{array}\right) / N$ !. In the physics literature the corresponding ensemble appears as a Coulomb gas of $N$ unit-charge particles (i.e a one component plasma) confined to the sphere in a neutralizing uniform background $\omega$ (see for example [27]). More generally, on any Riemann surface of genus $g$ the bosonization formula [1] gives

$$
\left\|\operatorname{det} S^{(k)}\right\|\left(x_{1}, \ldots x_{N}\right)=Z_{N} \exp \left(-\sum_{i \neq j} G\left(x_{i}, x_{j}\right)+r\left(x_{1}, \ldots, x_{N}\right)\right)
$$

where $G$ is the Green function of the Laplacian induced by the metric $\omega_{0}$ and where the second term $r$ appearing above vanishes for genus $g=0$, while for $g>0$ it may be expressed in terms of the Riemann theta function on the Jacobian torus of the Riemann surface $X$ (giving a contribution which is lower order than the first term; see [56] and references therein). However, when $n>1$ it should be stressed that there is no tractable formula for $\left\|\operatorname{det} S^{(k)}\right\|\left(x_{1}, \ldots x_{N}\right)$, even to the leading order.

When $\beta_{k}=k$ the probability measure $\mu^{\left(N_{k}, \beta_{k}\right)}$ in formula 5.11defines a determinantal point process i.e. its density can be written as

$$
\left\|\operatorname{det}_{i, j \leq N}\left(K^{(k)}\left(x_{i}, x_{j}\right)\right)\right\| / N_{k} !
$$

where $K^{(k)}(x, y)$ denotes the kernel of the orthogonal projection onto the space $H^{0}(X, k L)$ viewed as a subspace of the space $C^{\infty}(X, k L)$ of all smooth sections equipped with the $L^{2}$-norm determined by $(\|\cdot\|, d V)[5,39]$.

The following result generalizes the LDP in [5] for determinantal point processes (or more generally for the case $\beta=\infty$ ) to the general case where $\left.\left.\beta_{k} \rightarrow \beta \in\right] 0, \infty\right]$ :

Theorem 5.7. Let $(X, L)$ be a polarized manifold and assume given the data $\left(\|\cdot\|, d V, \beta_{k}\right)$ consisting of a Hermitian metric $\|\cdot\|$ on $L$, a volume form $d V$ on $X$ and a sequence of positive number $\left.\left.\beta_{k} \rightarrow \beta \in\right] 0, \infty\right]$. Then the law of the empirical 
measures $\delta_{N_{k}}$ of the corresponding deformed determinantal point processes with $N_{k}$ particles satisfies a LDP with speed $\beta_{k} N_{k}$ and rate functional

$$
F_{\beta}(\mu)=E_{\omega_{0}}(\mu)+\frac{1}{\beta} D_{d V}(\mu)-C_{\beta},
$$

where $E_{\omega_{0}}(\mu)$ is the pluricomplex energy of $\mu$ with respect to the curvature form $\omega_{0}$ of $\|\cdot\|$ and

$$
C_{\beta}=\inf _{\mathcal{M}_{1}(X)} F_{\beta}=-\lim _{N \rightarrow \infty} \frac{1}{N_{k} \beta_{k}} \log Z_{N . \beta_{k}},
$$

In particular, $\delta_{N_{k}}$ converges in law to the deterministic measure given by the unique minimizer $\mu_{\beta}$ of $F_{\beta}$. Moreover, when $\beta<\infty$ the measure $\mu_{\beta}$ is the normalized volume form $\omega_{\beta}$ of the twisted Kähler-Einstein metric corresponding to the twisting form $\eta:=$ $\beta \omega_{0}+R i c d V$.

In fact, the Kähler form $\omega_{\beta}$ may be recovered directly from the limiting volume form $\mu_{\beta}$ by differentiation twice (as follow from the very definition of the twisted Kähler-Einstein equation 5.2):

$$
\omega_{\beta}:=\frac{i}{2 \pi} \frac{1}{\beta} \partial \bar{\partial} \log \frac{\mu_{\beta}}{d V}+\omega_{0},
$$

Using basic compactness properties of the space $P S H\left(X, \omega_{0}\right)$ one then arrives at the following corollary (see [9] for the proof):

Corollary 5.8. Given data as in the previous theorem with $\beta \in] 0, \infty[$, the following sequence of Kähler forms on $X$

$$
\omega^{(k)}:=d d^{c} \frac{1}{\beta} \log \frac{\int_{X^{N_{k}-1}}\left\|\operatorname{det} S^{(k)}\left(\cdot, x_{2}, \ldots x_{N_{k}}\right)\right\|^{2 \beta / k} d V^{\otimes\left(N_{k}-1\right)}}{d V}+\omega_{0},
$$

converges to the unique solution $\omega_{\beta}$ of the twisted Kähler-Einstein metric corresponding to the twisting form $\eta:=\beta \omega_{0}+$ Ricd $V$.

Remark 5.9. The previous corollary yields a quasi-explicit way of approximating the solution $\omega_{\beta}$ to the twisted KE equation in question (or equivalently the solution $\varphi_{\beta}$ of the corresponding complex Monge-Ampère equation 5.4), by performing integrals over the spaces $X^{N_{k}-1}$ of increasing dimension. The procedure becomes explicit as soon as one has constructed bases in the spaces $H^{0}(X, k L)$, for $k$ sufficiently large.

5.2.1. The canonical random point processes on $X$. We start by recalling the basic fact that, by the very definition of the canonical line bundle $K_{X}$, any holomorphic section $s_{k}$ of the $k$ th tensor power of $K_{X}$ (i.e. $s_{k} \in H^{0}\left(X, k K_{X}\right)$ induces a measure on $X$, symbolically denoted by $\left(s_{k} \wedge \bar{s}_{k}\right)^{1 / k}$. Concretely, given an open set $U \subset X$ with holomorphic coordinates $\left(z_{1}, \ldots, z_{n}\right)$ and writing $s_{k \mid U}=f_{k} d z^{\otimes k}$ for a holomorphic function $f_{k}$ on $U$, where $d z:=d z_{1} \wedge \cdots \wedge d z_{n}$ trivializes $K_{X}$ over $U$,

$$
\left(s_{k} \wedge \bar{s}_{k}\right)_{\mid U}^{1 / k}=\left|f_{k}\right|^{2 / k} i^{n^{2}} d z \wedge d \bar{z},
$$

which is independent of $U$ and thus defines a global measure on $X$ (using any holomorphic atlas on $X$ ). We also recall that any volume form $d V$ on $X$ induces a metric 
$\|\cdot\|_{d V}$ on the canonical line bundle $K_{X}$ with the property that, if $s_{k} \in H^{0}\left(X, k K_{X}\right)$ then $\left(s_{k} \wedge \bar{s}_{k}\right)_{\mid U}^{1 / k}$ may be expressed as

$$
\left(s_{k} \wedge \bar{s}_{k}\right)_{\mid U}^{1 / k}=\left\|s_{k}\right\|_{d V}^{2 / k} d V
$$

as follows immediately from the definitions.

Now, fixing a volume form $d V$ on $X$ we can apply the relation 5.15 to $X^{N}$ equipped with the induced volume form $d V^{\otimes N}$ and the corresponding metric on $L$ and deduce that the canonical probability measure $\mu^{\left(N_{k}\right)}$ on $X^{N_{k}}$ defined by formula 1.6 coincides with the probability measured in formula 5.11 corresponding to the data $\left(\|\cdot\|_{d V}, d V, 1\right)$ Hence, Theorem 1.2 is indeed a special case of Theorem 5.7 (also using that $\eta=0$ for this particular data).

5.3. Proof of Theorem 5.7. To apply Theorem 1.1 in the present setting first note that the Hamiltonian is given by

$$
E^{\left(N_{k}\right)}\left(x_{1}, x_{2}, \ldots x_{N_{k}}\right):=-\frac{1}{k} \log \left\|\left(\operatorname{det} S^{(k)}\right)\left(x_{1}, x_{2}, \ldots x_{N_{k}}\right)\right\|^{2},
$$

where $\operatorname{det} S^{(k)}$ is defined by formula 5.13. The validity of the first assumption in Theorem 1.1 is then a consequence of the following result from [12], where $\beta_{N_{k}}=k$ :

Theorem 5.10. [12]. Let $L \rightarrow X$ be a positive line bundle equipped with a smooth Hermitian metric $\|\cdot\|$ on $L$ with curvature form $\omega_{0}$ and $d V$ a volume form on $X$. Then

$$
\lim _{k \rightarrow \infty}-\frac{1}{k N_{k}}\left(\log \int_{X^{N_{k}}}\left\|\operatorname{det} S^{(k)}\right\|^{2}\left(x_{1}, \ldots, x_{N}\right) e^{-k u\left(x_{1}\right)-\cdots-k u\left(x_{N}\right)}\right)=\mathcal{F}(u),
$$

where $\mathcal{F}$ is the Gateaux differentiable functional defined by formula 5.9

To verify the second assumption in Theorem 1.1, concerning quasi-superharmonicity, we first observe that we may as well assume that $d V$ is the volume form $d V_{g}$ of the metric $g$ defined by the Kähler form $\omega_{0}$. Indeed, $d V=e^{-u \beta} d V_{g}$ for some smooth function $u$ and hence changing $d V$ corresponds to changing the metric $\|\cdot\|$ to $\|\cdot\| e^{-u / 2}$. Next, we recall that, in general, $\log \|s\|^{2}$ is $k \omega-$ psh for any holomorphic section $s$ of $k L \rightarrow X$ (where $\omega$ is the curvature form of $\|\cdot\|$ ). Hence, we get,

$$
\Delta_{g} \log \|s\|^{2 / k} \geq-\lambda
$$

for some positive constant $\lambda$. Applying the latter inequality to $\| \operatorname{det}\left(s^{(k)}\left(\cdot, x_{2}, \ldots, x_{N}\right) \|\right.$ for $x_{2}, \ldots, x_{N}$ thus shows that Theorem 1.1 can be applied to get the LDP in Theorem 5.7 .

Next, we will show that the unique minimizer $\mu_{\beta}$ of the rate functional $F_{\beta}$ appearing in Theorem 1.1 coincides with the normalized volume form $\omega_{\beta}$ of the corresponding twisted Kähler-Einstein metric, by applying the general Lemma 3.12. It should however be stressed that while the infimum in the left hand side of formula 3.9 is always attained at some $\mu_{0} \in \mathcal{M}_{1}(X)$ (by weak compactness and lower-semi continuity) this is not so for the right hand side, in general. But in the present setting the sup is attained, when $L$ is assumed to be positive, thanks to the Aubin-Yau theorem. Indeed, first setting

$$
g(u)=\beta^{-1} \log \int e^{\beta u} d V,
$$


for a given $\beta \in] 0, \infty\left[\right.$ gives $g^{*}(\mu)=\beta^{-1} D_{d V}(\mu)$ if $\mu \in \mathcal{M}_{1}(X)$ and $g^{*}(\mu)=\infty$ otherwise, as is well-known [30] (and follows from Jensen's inequality applied to the $\log )$. Moreover, by the dominated convergence theorem

$$
d g_{\mid u}=\frac{e^{\beta u} d V}{\int_{X} e^{\beta u} d V} \in \mathcal{M}_{1}(X)
$$

Letting $\mathcal{F}$ be the functional on $C^{0}(X)$ defined by formula 5.9 the critical point Eq. 3.10 thus becomes

$$
M A(P u)=\frac{e^{\beta u} d V}{\int_{X} e^{\beta u} d V},
$$

when $u$ is smooth, say. Up to replacing $u$ by $u+C$ we may as well assume that the denominator above is equal to 1 . In particular, when $u \in \mathcal{H}(X, \omega)$ the equation above is precisely the Aubin-Yau equation 5.4, which, by the Aubin-Yau theorem admits a (unique) solution $u_{\beta} \in \mathcal{H}(X, \omega)$. Hence, by the previous lemma $\mu_{\beta}:=M A\left(u_{\beta}\right)$ is the unique minimizer of the rate functional $F_{\beta}$ appearing in Theorem 1.1, in the present setting. Finally, as explained in Sect. 5.1.3 $\mu_{\beta}$ is the volume form of the Kähler form $\omega_{\beta}$ solving the twisted Kähler-Einstein equation 5.2.

Remark 5.11. To see the relation to the pluricomplex energy introduced in [14] we write, as in formula 3.11 ,

$$
f^{*}(\mu)=\sup _{u \in C^{0}(X)} \mathcal{E}(P u)-\langle u, \mu\rangle
$$

when $\mu \in \mathcal{M}_{1}(X)$, which coincides with the pluricomplex energy of $\mu$, with respect to $\omega_{0}$ in [14] (using the notation in [7]). More concretely, a direct calculation reveals that when $\mu$ is a volume form

$$
E(\mu)=\frac{1}{V} \sum_{j=0}^{n-1} \frac{1}{j+2} \int_{X} d \varphi_{\mu} \wedge d^{c} \varphi_{\mu} \wedge \frac{\left(d d^{c} \varphi_{\mu}+\omega_{0}\right)^{j}}{j !} \wedge \frac{\omega_{0}^{n-1-j}}{(n-1-j) !},
$$

where $\varphi_{\mu} \in \mathcal{H}\left(X, \omega_{0}\right)$ is the solution to the Calabi-Yau equation 1.13, which in Aubin's notation [3] means that $E(\mu)=c_{n}(I-J)\left(\varphi_{\mu}\right)$ (using [20] the formula above holds for any $\mu$ such that $E(\mu)<\infty$, by letting $\wedge$ denote the non-pluripolar products [20]). Thus $E(\mu)$ is a generalization of the classical Dirichlet energy on a Riemann surface. The relation $F_{\beta}\left(\omega^{n}\right)=\kappa(\omega)$, where $\kappa$ denotes the twisted version of Mabuchi's $K$ energy then follows from the Chen-Tian formula for the K-energy (see [7] and [9] for a direct proof using convex analysis). Moreover, the restriction to $\mathcal{H}\left(X, \omega_{0}\right)$ of the dual functional $f(-u)+g(u)$ appearing in Lemma 3.12 coincides with the Ding functional in Kähler geometry [7]. An alternative proof of the fact that $\omega_{\beta}^{n}$ minimizes $F_{\beta}$ on $\mathcal{M}_{1}(X)$ can then be given by using that $\omega_{\beta}$ is a critical point of $\kappa$ and hence, by convexity, minimizes $\kappa$ on $\mathcal{H}\left(X, \omega_{0}\right)$. Accordingly, the Calabi-Yau isomorphism $\omega \mapsto \omega^{n}$ shows that $\omega_{\beta}^{n}$ minimizes the restriction of $F_{\beta}$ to the subspace of all volume forms in $\mathcal{M}_{1}(X)$. However, showing that the infimum of $F_{\beta}$ over all of $\mathcal{M}_{1}(X)$ coincides with the infimum over the subspace of volume forms requires the following non-trivial fact: any $\mu$ such that $E(\mu)<\infty$ can be written as a weak limit of volume forms $\mu_{j}$ such that $E\left(\mu_{j}\right) \rightarrow E(\mu)$ and $D_{d V}\left(\mu_{j}\right) \rightarrow D_{d V}(\mu)$ (see [16] where more general results are obtained). 
5.4. The generalization to big line bundles and varieties of positive Kodaira dimension. Let us briefly give some indications about the extension of Theorem 5.7 to line bundles $L$ which are merely assumed big, established in the companion paper [9]. In analytic terms $L$ is big iff $c_{1}(L)$ contains a positive current on $X$ which is strictly positive in the sense that it is bounded from below by a Kähler form. However, in general, there is a proper open subset $\Omega \subset X$ such that all positive currents in $c_{1}(L)$ are equal to $-\infty$ on the complement $X-\Omega$ (which can be taken to be a complex subvariety of $X$ ). Fixing a reference smooth Hermitian metric $\|\cdot\|$ on $L$ with curvature form $\omega_{0}$ in $c_{1}(L)$ the space of positive currents in $c_{1}(L)$ gets identified, as before, with the space $P S H\left(X, \omega_{0}\right)$ of all $\omega_{0}-$ psh functions, modulo constants (however, in general all elements in $P S H\left(X, \omega_{0}\right)$ will be singular along the subvariety $X-\Omega$ ). Moreover, the non-pluripolar MongeAmpère operator can be defined on $\operatorname{PSH}\left(X, \omega_{0}\right)$, by restricting to $\Omega$ [20]. Then the functional $\mathcal{F}$ can be defined essentially as before and Theorem5.7 still holds (again using [12] to verify the first assumption in Theorem 1.1) Invoking, the general Theorem 1.1 thus establishes an LDP with a rate functional $F_{\beta}$, admitting a unique minimizer $\mu_{\beta}$ as before. However, one new difficulty is to show that $\mu_{\beta}$ can be written as $M A\left(\varphi_{\beta}\right)$ for the solution to the Eq. 5.4 with minimal singularities, whose existence is provided by the general results in $[14,20]$. The problem is that Lemma 3.12 cannot be applied as it is not clear that $\varphi_{\beta}$ is of the form $P u$ for some $u$ in $C^{0}(X)$ (even if $u$ can be taken to be in $L^{\infty}(X)$ ). But using the variational calculus in [7,14] shows that $\mu_{\beta}$ is of the desired form.

In particular, when $K_{X}$ is big, i.e. $X$ is a variety of general type, the corresponding positive current $\omega_{\beta}$ is the canonical Kähler-Einstein current in $X$ [14,20]. In the general case of a variety of positive Kodaira dimension $\kappa \leq n$ (where $\kappa=n$ iff $K_{X}$ is big) one can use the Ithaka fibration $X \rightarrow Y$ to represent $K_{X}$ as the pull-back of a big line bundle $L$ on the $\kappa$-dimensional manifold $Y$. Using the Fujino-Mori canonical bundle formula this reduces the proof of the convergence on $X$ to the application of a generalization of Theorem 5.7 concerning big line bundles on $Y$ endowed with a singular volume form $d V$. As shown in [9] this realizes the corresponding canonical limiting current $\omega_{\beta}$ as the pull-back to $X$ of a (singular) Kähler form on $Y$ solving a twisted Kähler-Einstein equation of the form 5.2, where $\eta$ is a current on $Y$ determined by the geometry of $X$ (the canonical current $\omega_{\beta}$ first appeared in a different geometric context in [51,52]).

\section{Outlook}

6.1. $\beta=0$. Let $(X, L)$ be a polarized manifold and fix a Kähler metric $\omega_{0}$ in $c_{1}(L)$. By Corollary 5.8 (and well-known stability properties of the complex Monge-Ampère operator) one can recover the unique (normalized) smooth solution to the Calabi-Yau equation

$$
\left(\omega_{0}+i \partial \bar{\partial} \varphi\right)^{n}=d V
$$

[53] as the double limit $\varphi:=\lim _{\beta \rightarrow \infty} \lim _{k \rightarrow \infty} \varphi_{\beta}^{(k)}$, where

$$
\varphi_{\beta}^{(k)}:=\frac{1}{\beta} \log \frac{\int_{X^{N_{k}-1}}\left\|\operatorname{det} S^{(k)}\left(\cdot, x_{2}, \ldots x_{N_{k}}\right)\right\|^{2 \beta / k} d V^{\otimes\left(N_{k}-1\right)}}{d V}-\log Z_{N}
$$

Formally interchanging the two limits thus suggests the following 
Conjecture 6.1. Let $(X, L)$ be a polarized manifold and $\omega_{0}$ a Kähler metric in $c_{1}(L)$. Then the unique smooth solution $\varphi$ to the Calabi-Yau equation 6.1, normalized so that $\int_{X} \varphi d V=0$, may be represented as the following limit in $L^{1}(X)$ :

$$
\varphi:=\lim _{k \rightarrow \infty} \varphi^{(k)}, \quad \varphi^{(k)}:=\frac{1}{k} \frac{\int_{X^{N_{k}-1}} \log \left\|\operatorname{det} S^{(k)}\left(\cdot, x_{2}, \ldots x_{N_{k}}\right)\right\|^{2} d V^{\otimes\left(N_{k}-1\right)}}{d V}-C_{k},
$$

where the constant $C_{k}$ ensures that $\int_{X} \varphi^{(k)} d V=0$.

The conjectural formula above can be seen as a generalization to the non-linear complex Monge-Ampère operator of the classical Green's formula for the solution of the Poisson equation for the Laplacian on a Riemann surface. Indeed, when $X$ is a Riemann surface the limit $\varphi$ above is precisely given by the Green formula in question (as follows from the bosonization formula 5.14). It turns out that the validity of the conjecture above would follow from the existence of the corresponding mean energy $\bar{E}(\mu)$, for any volume form $\mu$ (see Problem 4.10). This is shown precisely as in the setting of the real Monge-Ampère operator considered in $[8,40]$ where the analog of the previous conjecture was established using permanents as a replacements of the determinants appearing in the present setting. In particular, when $X$ is a Calabi-Yau manifold, i.e., $K_{X}$ is trivial, the conjecture would imply a quasi-explicit formula for the unique Ricci flat Kähler metric $\omega \in c_{1}(L)$, i.e., solving the Kähler-Einstein equation with vanishing cosmological constant, $\Lambda=0$.

6.2. $\beta<0$. By Lemma 4.11 the existence of the mean energy (and thus the resolution of the conjecture above) would follow if one could establish the asymptotics in formula 4.6 of the corresponding partition functions $Z_{N_{k}, \beta / k}$ (assumed finite) for all $\beta>\beta_{0}$, for some negative number $\beta_{0}$. It can be shown that $Z_{N, \beta_{N}}$ is indeed finite for some negative $\beta_{0}$, sufficiently close to zero. In fact, both sides of formula 4.6 are finite when $\beta>\beta_{0}$ (where the critical negative $\beta_{0}$ depends on $(X, L)$ ). This motivates the following

Conjecture 6.2. Let $(X, L)$ be a polarized manifold and assume given the data $(\|\cdot\|, d V)$ consisting of a Hermitian metric $\|\cdot\|$ on $L$, a volume form $d V$ on $X$. For a given negative number $\beta_{0}$ the following is equivalent:

- For any $\beta>\beta_{0}$ the partition functions $Z_{N_{k}, \beta}$ are finite for $k$ sufficiently large

- For any $\beta>\beta_{0}$ the functional $\beta F_{\beta}$ admits a minimizer on $\mathcal{M}_{1}(X)$

- For any $\beta>\beta_{0}$ the measures $\left(\delta_{N}\right)_{*}\left(e^{-\beta H^{\left(N_{k}\right)}} d V^{\otimes N_{k}}\right)$ on $\mathcal{M}_{1}(X)$ satisfy a LDP with speed $N$ and rate functional

$$
\beta F_{\beta}(\mu)=\beta E_{\omega_{0}}(\mu)+D_{d V}(\mu),
$$

where $E_{\omega_{0}}(\mu)$ is the pluricomplex energy of $\mu$ with respect to the curvature form $\omega_{0}$ of $\|\cdot\|$.

In particular, if the conjectural LDP above holds then the functional $\beta F_{\beta}$ is lower semi-continuous and the large $N$-limit of the laws of $\delta_{N_{k}}$ for the corresponding random point processes is concentrated on the (non-empty) set of minimizers of $\beta F_{\beta}$. By [7] any such minimizer is the volume form of a Kähler metric $\omega_{\beta}$ solving the twisted KählerEinstein equation 5.2 corresponding to the data $\left(\omega_{0}, d V, \beta\right)$ and $\beta F_{\beta}$ may be identified with the corresponding twisted K-energy functional. Moreover, if the LDP holds then 
it follows that $Z_{N_{k}, \beta} \leq C_{\beta}^{N}$, when $\beta>\beta_{0}$. The conjecture should be contrasted with the fact that, in general, $\beta F_{\beta}$ is unbounded from below if $\beta$ is sufficiently negative and even when $\beta F_{\beta}$ is bounded from below there exist, in general, twisted Kähler-Einstein metrics whose volume forms do not minimize $\beta F_{\beta}$.

In the case when $L$ is the dual $-K_{X}$ of the canonical line bundle, i.e., $X$ is a Fano manifold (which equivalently means that $\eta$ can be taken to be zero) the equivalence between the first two points in the conjecture above can be seen as a probabilistic analog of the Yau-Tian-Donaldson conjecture saying that a Fano manifold $X$ admits a KählerEinstein metric with positive Ricci curvature ((i.e. $\Lambda>0$ ) iff $X$ is $\mathrm{K}$-stable in the algebro-geometric sense; see the companion paper [9] for more detailed explanations of these relations.

Interestingly, the notion of negative temperature has already appeared in Onsager's work on the 2D vortex model [46]. Using the bosonization formula 5.14 on a Riemann surface and large $N$-results for vortex models (as in $[22,26,41]$ ) it can be shown that the conjecture above holds when $X$ is a Riemann surface. Moreover, then the critical $\beta_{0}$ is equal to 2 , when the volume (degree) of $L$ is normalized to be one. In our normalizations this corresponds to the critical negative temperature $8 \pi$ in the vortex model $[26,41]$ (a detailed proof of this will appear elsewhere).

Acknowledgements. It is a pleasure to thank Sebastien Boucksom, David Witt-Nyström, Vincent Guedj and Ahmed Zeriahi for the stimulating collaborations [12-14], which paved the way for the present work. I am also grateful to Bo Berndtsson for infinitely many fruitful discussions on complex analysis and Kähler geometry over the years. Thanks, in particular, to Sebastien Boucksom for illuminating discussions on Lemma 3.6. Also thanks to the referee for comments that helped to improve the presentation of the paper.The present paper, together with the companion paper [9], supersedes the first arXiv version of the paper [9]. This work was supported by grants from the ERC and the KAW foundation.

Open Access This article is distributed under the terms of the Creative Commons Attribution 4.0 International License (http://creativecommons.org/licenses/by/4.0/), which permits unrestricted use, distribution, and reproduction in any medium, provided you give appropriate credit to the original author(s) and the source, provide a link to the Creative Commons license, and indicate if changes were made.

\section{Appendix: The Constant in the Cheng-Yau Gradient Estimate}

Set $\phi:=|\nabla u / u|$ and $F:=\phi\left(a^{2}-\rho^{2}\right)$, where $\rho \leq a \leq 1$. We will follow the exposition in [47]. First, Bochner's identity gives after some calculations that, for any $x$,

$$
\frac{\Delta \phi}{\phi} \geq \frac{\phi^{2}}{(n-1)}-(n-1) k^{2}-\left(2-\frac{2}{(n-1)}\right) \frac{\nabla \phi}{\phi} \cdot \frac{\nabla u}{u}
$$

(see the bottom of [47, page 141]). Let now $x_{1}$ be a point in the interior of $B_{a}\left(x_{0}\right)$ where $F$ attains it maximum and assume that $\rho\left(:=d\left(x, x_{0}\right)\right)$ is smooth close to $x_{1}$. Next $\nabla F=0$ at $x_{1}$ gives

$$
\frac{\nabla \phi}{\phi}=\frac{\nabla \rho^{2}}{a^{2}-\rho^{2}}=\frac{2 \rho \nabla \rho}{a^{2}-\rho^{2}}
$$

(in the following all (in-)equalities are evaluated at $x=x_{1}$ ) and $\Delta F \leq 0$ at $x_{1}$ gives

$$
\frac{\Delta \phi}{\phi}-\frac{\Delta \rho^{2}}{a^{2}-\rho^{2}}-\frac{2\left|\nabla \rho^{2}\right|^{2}}{\left(a^{2}-\rho^{2}\right)^{2}} \leq 0
$$


Now, by the Laplacian comparison

$$
\Delta \rho^{2} \leq 2+2(n-1)(1+k \rho)
$$

Substituting this into the previous inequality we get (using $|\nabla \rho| \leq 1$ )

$$
\frac{\Delta \phi}{\phi}-\frac{2+2(n-1)(1+k \rho)}{a^{2}-\rho^{2}}-\frac{8 \rho^{2}}{\left(a^{2}-\rho^{2}\right)^{2}} \leq 0
$$

By 7.2

$$
-\frac{\nabla \phi}{\phi} \cdot \frac{\nabla u}{u} \geq-\frac{2 \rho \phi}{a^{2}-\rho^{2}}
$$

Hence, Eq. 7.1 combined with Eqs. 7.3 and the previous inequality gives

$0 \geq \frac{\phi^{2}}{(n-1)}-(n-1) k^{2}-\frac{4(n-2)}{(n-1)} \frac{2 \rho \phi}{a^{2}-\rho^{2}}-\frac{(2+2(n-1))(1+k \rho)}{a^{2}-\rho^{2}}-\frac{8 \rho^{2}}{\left(a^{2}-\rho^{2}\right)^{2}}$,

Equivalently, multiplying by $\left(a^{2}-\rho^{2}\right)^{2}$ gives

$$
0 \geq \frac{F^{2}}{(n-1)}-(n-1) k^{2}\left(a^{2}-\rho^{2}\right)^{2}-\frac{4(n-2)}{(n-1)} 2 \rho F-(2+2(n-1))(1+k \rho)\left(a^{2}-\rho^{2}\right)-8 \rho^{2},
$$

Since we are only interested in the large $n$ behaviour (and $0 \leq \rho \leq a$ ) we deduce from the previous inequality that

$$
0 \geq \frac{F^{2}}{(n-1)}-8 \rho F-n k^{2}\left(a^{2}\right)^{2}-2 n(1+k) a^{2}-8 \rho^{2}
$$

giving, after multiplication by $n$,

$$
0 \geq F^{2}-8 a n F-n^{2} k^{2}\left(a^{2}\right)^{2}-2 n^{2}(1+k) a^{2}-8 a^{2} n,
$$

which we write as

$$
(4 a n)^{2}+n^{2} k^{2}\left(a^{2}\right)^{2}+2 n^{2}(1+k) a^{2}+8 a^{2} n \geq(F-4 a n)^{2},
$$

giving

$$
a^{2} n^{2}\left(26+k^{2} a^{2}+2 k\right) \geq(F-4 a n)^{2}
$$

Hence

$$
a n\left(\left(26+k^{2} a^{2}+2 k\right)^{1 / 2}+4\right) \geq F:=\phi(a-\rho)(a+\rho) \geq \phi(a-\phi) a,
$$

so that

$$
n\left(\left(26+k^{2} a^{2}+2 k\right)^{1 / 2}+4\right) \geq \phi(a-\phi),
$$

This shows that, if $a \leq 1$, there exists a constant $C$, only depending on an upper bound on $k$, such that

$$
C n \geq \phi(a-\phi),
$$

as desired. 


\section{References}

1. Alvarez-Gaumé, L., Bost, J.-B., Moore, G., Nelson, P., Vafa, C.: Bosonization on higher genus Riemann surfaces. Commun. Math. Phys. 112(3), 503-552 (1987)

2. Arnaudon, M., Driver, B.K., Thalmaier, A.: Gradient estimates for positive harmonic functions by stochastic analysis. Stoch. Process. Appl. 117(2), 202-220 (2007)

3. Aubin, T.: Equations du type Monge-Ampère sur les variètès Kählériennes compactes. Bull. Sci. Math. (2) 102(1), 63-95 (1978)

4. Bardenet, R., Hardy, A.: Monte Carlo with Determinantal Point Processes. arXiv:1605.00361

5. Berman, R.J.: Determinantal point processes and fermions on complex manifolds: large deviations and Bosonization. Commun. Math. Phys. 327(1), 1-47 (2014)

6. Berman, R.J.: Kahler-Einstein metrics emerging from free fermions and statistical mechanics. J. High Energy Phys. (JHEP) 2011(10), 22 (2011)

7. Berman, R.J.: A thermodynamical formalism for Monge-Ampere equations, Moser-Trudinger inequalities and Kahler-Einstein metrics. Adv. Math. 248, 1254 (2013)

8. Berman, R.J.: Statistical mechanics of permanents, real-Monge-Ampere equations and optimal transport. arXiv: 1302.4045

9. Berman, R.J.: Kähler-Einstein metrics, canonical random point processes and birational geometry. In: AMS Proceedings of the 2015 summer research institute on algebraic geometry. arXiv:1307.3634 (2015, to appear)

10. Berman, R.J.: From Monge-Ampere equations to envelopes and geodesic rays in the zero temperature limit. arXiv: 1307.3008

11. Berman, R.J.: On large deviations for Gibbs measures, mean energy and Gamma convergence. arXiv: 1610.08219

12. Berman, R.J., Boucksom, S.: Growth of balls of holomorphic sections and energy at equilibrium. Invent. Math. 181(2), 337 (2010)

13. Berman, R.J., Boucksom, S., Witt Nyström, D.: Fekete points and convergence towards equilibrium measures on complex manifolds. Acta Math. 207(1), 1-27 (2011)

14. Berman, R.J., Boucksom, S., Guedj, V., Zeriahi, A.: A variational approach to complex Monge-Ampere equations. Publ. math. de l'IHÉS, 1-67 (2012)

15. Berman, R.J., Onnheim, M.: Propagation of chaos, Wasserstein gradient flows and toric Kahler-Einstein metrics. arXiv:1501.07820

16. Berman, R.J., Darvas, T., Lu, C.H.: Convexity of the extended K-energy and the large time behaviour of the weak Calabi flow. Geom. Topol. (to appear). arXiv:1510.01260

17. Ben Arous, G., Guionnet, A.: Large deviations for Wigner's law and Voiculescu's non-commutative entropy. Probab. Theory Relat. Fields 108(4), 517-542 (1997)

18. Ben Arous, G., Zeitouni, O.: Large deviations from the circular law. ESAIM Probab. Stat. 2, 123134 (1998)

19. Bloom, T., Levenberg, N.: Pluripotential energy and large deviation. Indiana Univ. Math. J. 62(2), 523550 (2013)

20. Boucksom, S., Essidieux, P., Guedj, V. Zeriahi: Monge-Ampere equations in big cohomology classes. Acta Math. 205(2), 199-262 (2010)

21. Borzellino, J.E., Zhu, S.-H.: The splitting theorem for orbifolds. Ill. J. Math. 38(4), 679-691 (1994)

22. Bodineau, T., Guionnet, A.: About the stationary states of vortex systems. Ann. Inst. Henri Poincaré Probab. Stat. 35, 205-237 (1999)

23. Braides, A.: $\Gamma$-Convergence for Beginners. Oxford University Press, Oxford (2002)

24. Brézis, H.: Functional Analysis, Sobolev Spaces and Partial Differential Equations. Springer, New York (2011)

25. Brøndsted, A., Rockafellar, R.T.: On the subdifferentiability of convex functions. Proc. Am. Math. Soc. 16, 605-611 (1965)

26. Caglioti, E., Lions, P.-L., Marchioro, C., Pulvirenti, M.: A special class of stationary flows for twodimensional Euler equations: a statistical mechanics description. Commun. Math. Phys. 143(3), 501525 (1992)

27. Caillol, J.M.: Exact results for a two-dimensional one-component plasma on a sphere. J. Phys. 42(12), L245-L-247 (1981)

28. Chafaï, D., Gozlan, N., Zitt, P.-A.: First-order global asymptotics for confined particles with singular pair repulsion. Ann. Appl. Probab. 24(6), 2371-2413 (2014)

29. Cheng, S.Y., Yau, S.T.: Differential equations on Riemannian manifolds and their geometric applications. Commun. Pure Appl. Math. 28(3), 333-354 (1975)

30. Dembo, A., Zeitouni, O.: Large Deviation Techniques and Applications. Corrected Reprint of the Second (1998) Edition. Stochastic Modelling and Applied Probability, 38. Springer, Berlin (2010) 
31. Doran, C., Headrick, M., Herzog, C.P., Kantor, J.: Numerical Kaehler-Einstein metric on the third del Pezzo. Commun. Math. Phys. 282(2), 357-393 (2008)

32. Donaldson, S.K.: Some numerical results in complex differential geometry. Pure Appl. Math. Q. 5(2) (2009), Special Issue: In Honor of Friedrich Herzebruch. Part 1, 571-618

33. Dupuis, P., Laschos, V., Ramanan, K.: Large deviations for empirical measures generated by Gibbs measures with singular energy functionals. arXiv:1511.06928

34. Ellis, R.S., Haven, K., Turkington, B.: Large deviation principles and complete equivalence and nonequivalence results for pure and mixed ensembles. J. Stat. Phys. 101(5-6), 999-1064 (2000)

35. Eyink, G.L., Sreenivasan, K.R.: Onsager and the theory of hydrodynamic turbulence. Rev. Modern Phys. 78(1), 87-135 (2006)

36. Ferrari, F., Klevtsov, S., Zelditch, S.: Random Kähler metrics. Nucl. Phys. B 869(1), 89-110 (2013)

37. Fine, J.: Constant scalar curvature Kähler metrics on fibred complex surfaces. J. Differ. Geom. 68(3), 397$432(2004)$

38. Huichun, Z., Xiping, Z.: On a new definition of Ricci curvature on Alexandrov spaces. Acta Math. Sci. 30(6), 1949-1974 (2010)

39. Hough, J.B., Krishnapur, M., Peres, Y.1., Virág, B.: Determinantal processes and independence. Probab. Surv. 3, 206-229 (2006)

40. Hultgren, J.: Permanental Point Processes on Real Tori, Theta Functions and Monge-Ampère Equation. arXiv:1604.05645

41. Kiessling, M.K.H.: Statistical mechanics of classical particles with logarithmic interactions. Commun. Pure Appl. Math. 46, 27-56 (1993)

42. Kiessling, M.K.H.: Statistical mechanics approach to some problems in conformal geometry. Phys. A Stat. Mech. Appl. 279(1-4), 353-368 (2000)

43. Klevtsov, S.: Geometry and large N limits in Laughlin states. arXiv:1608.02928

44. Li, P., Schoen, R.: Lp and mean value properties of subharmonic functions on Riemannian manifolds. Acta Math. 153(3-4), 279-301 (1984)

45. Messer, J., Spohn, H.: Statistical mechanics of the isothermal Lane-Emden equation. J. Stat. Phys. 29(3), 561-578 (1982)

46. Onsager, L.: Statistical hydrodynamics. Supplemento Al Nuovo Cimento 6, 279-287 (1949)

47. Schoen, R.: Lecture 1 and 2 in "nonlinear partial differential equations in differential geometry". In: Hardt R., Wolf M. (eds.) IAS/Park City Math. Series. vol. 2 (1996)

48. Serfaty, S.: Coulomb gases and Ginzburg-Landau vortices. Zurich Lectures in Advanced Mathematics. European Mathematical Society (EMS), Zürich (2015)

49. Shiffman, B., Zelditch, S.: Distribution of zeros of random and quantum chaotic sections of positive line bundles. Commun. Math. Phys. 200(3), 661-683 (1999)

50. Sznitman, A.-S.: Topics in propagation of chaos. École d'Été de Probabilités de Saint-Flour XIX—1989, Lecture Notes in Math., 1464, pp. 165-251. Springer, Berlin (1991)

51. Song, Y., Tian, G.: Canonical measures and Kähler-Ricci flow. J. Am. Math. Soc. 25(2), 303-353 (2012)

52. Tsuji, H.: Canonical measures and the dynamical systems of Bergman kernels. Preprint arXiv:0805.1829 (2008)

53. Yau, S.-T.: On the Ricci curvature of a compact Kähler manifold and the complex Monge-Amp'ere equation. Commun. Pure Appl. Math. 31(3), 339-411 (1978)

54. Yau, S.T.: Non-linear analysis in geometry. Enseig. Math. (2) 33(1-2), 109-158 (1987)

55. Zeitouni, O., Zelditch, S.: Large deviations of empirical measures of zeros of random polynomials. Int. Math. Res. Not. IMRN 20, 3935-3992 (2010)

56. Zelditch, S.: Large deviations of empirical measures of zeros on Riemann surfaces. Int. Math. Res. Not. 2013(3), 592-664 (2012) 